DESY 93-174

TTP 93-41

December 1993

\title{
PROSPECTS OF MEASURING THE PARITY OF HIGGS PARTICLES
}

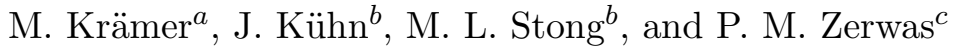 \\ ${ }^{a}$ Inst. Physik, Johannes Gutenberg-Universität, D-55099 Mainz FRG \\ ${ }^{b}$ Inst. Theor. Teilchenphysik, Univ. Karlsruhe, D-76128 Karlsruhe FRG \\ ${ }^{c}$ Deutsches Elektronen-Synchrotron DESY, D-22603 Hamburg FRG
}

\begin{abstract}
We analyze the prospects of measuring the parity of Higgs particles in the Standard Model and its supersymmetric extensions. Higgs decays are discussed in this context as well as production processes including, in particular, the fusion of Higgs particles in linearly polarized photon-photon collisions.
\end{abstract}




\section{Introduction}

While the Higgs particle in the Standard Model [1] must necessarily be a scalar particle, assigned the external quantum numbers $J^{P C}=0^{++}$, the Higgs spectrum in extended models such as supersymmetric theories includes also pseudoscalar $0^{-+}$states [2]. This non-trivial assignment of the quantum numbers invites the investigation of experimental opportunities to measure the parity of the Higgs states. This problem was approached in a general form quite early in Ref. [3] for Higgs decays into fermion and gauge boson pairs. It has recently been revived in a more specific form, and including production processes, for neutral particles in Refs. [4,5] and for charged particles in Ref. [6].

Spin correlations of fermions from Higgs decay (e.g., $\tau^{+} \tau^{-}$or $t \bar{t}$ ) are also sensitive to the parity of the Higgs [7]. This sensitivity is reflected in angular correlations of secondary decay products, in particular in the acollinearity distribution of $\pi^{+} \pi^{-}$from $\tau$ decays or $l^{+} l^{-}$from top decays.

Another interesting method is provided by the fusion of neutral Higgs particles [8 11] in linearly polarized photon-photon collisions [12]. Polarized high energy photon beams can be generated by Compton back-scattering of linearly polarized laser light [13]. The production of scalar particles requires parallel polarization of the two photons involved, while pseudoscalar particles require perpendicular polarization vectors [14.

Extracting the Higgs signal in $\gamma \gamma$ collisions is not an easy task since large numbers of $b \bar{b} / c \bar{c}$ and $W^{+} W^{-} / Z Z$ background events must be rejected [8, 15, 16]. However, the resolved $\gamma$ mechanisms do not pose background problems in the kinematical configurations relevant to asymmetry measurements. Choosing the laser energy only slightly higher than the Higgs threshold in the $\gamma \gamma$ collisions requires, the resolved $\gamma$ processes $\gamma g \rightarrow b \bar{b}$ and $g g \rightarrow b \bar{b}$ are strongly suppressed due to the steeply falling gluon spectrum. Background $\gamma \gamma, \gamma g, g g \rightarrow c \bar{c}$ charmed particle events must be suppressed by means of excellent $\mu$-vertex detectors.

\section{Neutral Higgs Decays to Fermion Pairs}

The most frequent fermion decay mode of neutral Higgs particles is in general the $b \bar{b}$ channel, Fig. 1. This applies in the Standard Model $(\mathcal{S M})$ [17,18] as well as its minimal supersymmetric extension $(\mathcal{M S S M})$ [19]. However, due to the depolarization effects in the fragmentation process, it is very difficult to extract information on the $b$ polarization state [20]. Much cleaner channels, though with branching ratios suppressed by an order of magnitude, are the $\tau$ and $t$ modes $\mathrm{U}$

$$
\begin{aligned}
& H, A \rightarrow \tau^{+} \tau^{-} \\
& H, A \rightarrow t \bar{t}
\end{aligned}
$$

\footnotetext{
${ }^{1}$ The generic notation $H$ will be used for the $0^{++}$Higgs particles, while $A$ will denote the pseudoscalar $0^{-+}$Higgs particles.
} 
The $\tau$ channel is useful in the $\mathcal{S} \mathcal{M}$ for Higgs masses less than $\sim 130 \mathrm{GeV}$, in supersymmetric theories generally over a much larger mass range [19]. Top quark decays are of interest in a wide range above the top threshold [21]. For large top masses, the top quarks decay, $t \rightarrow b W$, before fragmentation destroys the $t$-spin information [22]. The top quarks can thus be treated as undressed particles, like leptons.

Denoting the spin vectors of the fermion $f$ and the antifermion $\bar{f}$ in their respective rest frames by $s$ and $\bar{s}$, respectively, [the $\hat{z}$-axis oriented in the $f$ flight direction], the spin dependence of the decay probability is given by [4]

$$
\Gamma(H, A \rightarrow f \bar{f}) \sim 1-s_{z} \bar{s}_{z} \pm s_{\perp} \bar{s}_{\perp}
$$

This spin dependence translates directly into correlations among the fermion decay products. A few representative examples will be discussed in more detail.

\section{1. $\quad H, A \rightarrow \tau^{+} \tau^{-} \rightarrow \pi^{+} \bar{\nu} \pi^{-} \nu$}

Even though the decay mode $\tau^{ \pm} \rightarrow \pi^{ \pm} \nu_{\tau}\left(\bar{\nu}_{\tau}\right)$ is rare, it may serve as a simple example that illustrates the basic principles. Defining the polar angles between the $\pi^{ \pm}$and the $\tau^{-}$ direction in the $\tau^{ \pm}$rest frames by $\theta_{ \pm}^{*}$ and the relative azimuthal angle $\phi^{*}$ between the decay planes (Fig. 2), the angular correlation may be written [23]

$$
\frac{1}{\Gamma} \frac{d \Gamma\left(H, A \rightarrow \pi^{+} \bar{\nu} \pi^{-} \nu\right)}{d \cos \theta_{+}^{*} d \cos \theta_{-}^{*} d \phi^{*}}=\frac{1}{8 \pi}\left[1+\cos \theta_{-}^{*} \cos \theta_{+}^{*} \mp \sin \theta_{+}^{*} \sin \theta_{-}^{*} \cos \phi^{*}\right]
$$

Anticipating that the Higgs masses will be known very accurately before measurements of the parity will be carried out, the Higgs rest frame can in principle be reconstructed on an event-by-event basis in the Higgs-strahlung process $e^{+} e^{-} \rightarrow Z H$ and for associated production $e^{+} e^{-} \rightarrow Z A$. In practice, measurement errors and $\gamma$ radiation will smear out the reconstructed momenta of the final-state particles. Further complications occur in the reconstruction of the $\tau$ direction of flight, as the neutrinos are undetected. The angles $\theta_{ \pm}^{*}$ are related to the pion energies in the Higgs rest frame and they can therefore be measured, but a two-fold ambiguity remains in the $\tau$ direction and hence in the angle $\phi^{*}$. This ambiguity can in principle be resolved using the impact parameter between the pion momenta [24]. In our case, however, this procedure is unnecessary, as only $\cos \phi^{*}$ is required which may simply be extracted from the dot product of the two pion momenta in the Higgs rest frame:

$$
16 \vec{\pi}_{+} \cdot \vec{\pi}_{-}=m_{H}^{2}\left(\beta_{\pi} \cos \theta_{-}^{*}+\beta_{\tau}\right)\left(\beta_{\pi} \cos \theta_{+}^{*}-\beta_{\tau}\right)+4 m_{\tau}^{2} \sin \theta_{+}^{*} \sin \theta_{-}^{*} \cos \phi^{*}
$$

$\beta_{\tau}=\sqrt{1-4 m_{\tau}^{2} / m_{H}^{2}}$ and $\beta_{\pi}=\left(m_{\tau}^{2}-m_{\pi}^{2}\right) /\left(m_{\tau}^{2}+m_{\pi}^{2}\right)$ are the Higgs-to- $\tau$ and $\tau$-to-pion rest frame boosts, respectively. This allows us to reconstruct the distribution (3) directly.

A simple asymmetry in the azimuthal angle that projects out nicely the parity of the particle, can be derived [3, [1] by integrating out the polar angles

$$
\frac{1}{\Gamma} \frac{d \Gamma(H, A)}{d \phi^{*}}=\frac{1}{2 \pi}\left[1 \mp \frac{\pi^{2}}{16} \cos \phi^{*}\right]
$$


A useful observable sensitive to the parity of the decaying Higgs particle is the angle $\delta$ between the two charged pions in the Higgs rest frame [23]. The distribution of eqn.(3) is rewritten in terms of the angle $\delta$ through the substitution of the pion momenta in eqn.(勾):

$$
\begin{aligned}
16 \vec{\pi}_{+} \cdot \vec{\pi}_{-}=m_{h}^{2}[(1+ & \left.\left.\beta_{\tau} \beta_{\pi} \cos \theta_{-}^{*}\right)^{2}-16 m_{\pi}^{2} / m_{h}^{2}\right]^{1 / 2} \\
& \times\left[\left(1-\beta_{\tau} \beta_{\pi} \cos \theta_{+}^{*}\right)^{2}-16 m_{\pi}^{2} / m_{h}^{2}\right]^{1 / 2} \cos \delta
\end{aligned}
$$

The azimuthal angle $\phi^{*}$ can therefore be written as a function of the angles $\theta_{ \pm}^{*}$ and $\delta$ :

$$
\begin{aligned}
\frac{1}{\Gamma} \frac{d \Gamma(H, A)}{d \cos \theta_{-}^{*} d \cos \theta_{+}^{*} d \cos \delta} & =\frac{J}{8 \pi}\left[1+\cos \theta_{-}^{*} \cos \theta_{+}^{*} \mp \sin \theta_{+}^{*} \sin \theta_{-}^{*} \cos \phi^{*}\left(\theta_{+}^{*}, \theta_{-}^{*}, \delta\right)\right] \\
J & =\frac{8 \vec{\pi}_{+} \cdot \vec{\pi}_{-}}{m_{\tau}^{2} \cos \delta \sin \theta_{+}^{*} \sin \theta_{-}^{*} \sin \phi^{*}\left(\theta_{+}^{*}, \theta_{-}^{*}, \delta\right)}
\end{aligned}
$$

Integration over the polar angles $\theta_{ \pm}^{*}$ gives a rather complex function of $\delta$. Although the resulting distributions are very similar for most values of $\delta$, they behave differently in the limit $\delta \rightarrow \pi$. The scalar distribution approaches its maximum at $\delta=\pi$,

$$
\frac{1}{\Gamma_{H}} \frac{d \Gamma(H)}{d \cos \delta} \rightarrow \frac{2}{15} \frac{\left(5+\beta_{\tau}^{2}\right)}{\left(1-\beta_{\tau}^{2}\right)},
$$

while the pseudoscalar distribution peaks at a small but nonzero value of $\pi-\delta$, corresponding to a nonvanishing acollinearity angle between the pions. In the limit of vanishing pion mass, the distribution approaches zero as the pions are emitted back-to-back:

$$
\frac{1}{\Gamma_{A}} \frac{d \Gamma(A)}{d \cos \delta} \rightarrow(1+\cos \delta) \frac{1}{20} \frac{\left(5+10 \beta_{\tau}^{2}+\beta_{\tau}^{4}\right)}{\left(1-\beta_{\tau}^{2}\right)^{2}}
$$

Since the pion mass is very much smaller than the $\tau$ mass, the distributions for non-zero pion masses have much the same behavior in the limit of back-to-back pions, Fig. 3 .

The discussion of $\tau$ decays to multipion final states follows very much the same line. One may either treat the hadron system as a single particle with definite spin and variable mass $\sqrt{Q^{2}}$, or one may extract additional information from the individual pion momenta. The first strategy leads to a trivial generalization of the single pion case, the second will be detailed below.

Let us for definiteness consider the case of both $\tau^{\prime}$ 's decaying to $\rho(\widehat{=} 2 \pi)$. The correlation term in eqn.(3) is then reduced by the factor $\left(m_{\tau}^{2}-2 Q^{2}\right)^{2} /\left(m_{\tau}^{2}+2 Q^{2}\right)^{2}$. The kinematic relations must also be corrected by the mass of the hadron system $Q^{2}$ which can no longer be neglected relative to $m_{\tau}^{2}$. Predictions for the distribution of the acollinearity are shown in Fig. 4 for fixed $Q^{2}=m_{\rho}^{2}=(0.770 \mathrm{GeV})^{2}$.

The dilution factor, which is even more severe in the three-pion channel with $Q^{2} \approx m_{\tau}^{2} / 2$, can be circumvented by the analysis of the individual pion distributions. The direction of the pion momentum (defined in the $\tau$ rest frame) appears in eqn.(3) and replaces the spin 
vector $\vec{s}$ in eqn.(2). In the general case, $s(\bar{s})$ must be replaced by the vector $\pm R^{\mp} /\left(m_{\tau} \omega_{\mp}\right)$, where $R$ and $\omega$ are defined by 23]

$$
\begin{aligned}
\Pi_{\mu} & =4 \mathcal{R e}_{\mu} q \cdot J^{*}-2 q_{\mu} J \cdot J^{*} \\
\Pi_{5 \mu} & =2 \epsilon_{\mu \nu \rho \sigma} \mathcal{I}_{m} J^{\rho} J^{* \nu} q^{\sigma} \\
\omega & =p_{\mu}\left(\Pi^{\mu}-\gamma_{\mathrm{AV}} \Pi_{5}^{\mu}\right) \\
R_{\mu} & =\left(m_{\tau}^{2} g_{\mu \nu}-p_{\mu} p_{\nu}\right)\left(\gamma_{\mathrm{AV}} \Pi^{\nu}-\Pi_{5}^{\nu}\right)
\end{aligned}
$$

$q$ is the momentum of the neutrino, $p_{ \pm}$is the momentum of the $\tau^{ \pm}$and $J_{ \pm}$is the hadronic current, and $\gamma_{\mathrm{AV}}=2 g_{\mathrm{A}} g_{\mathrm{V}} /\left(g_{\mathrm{A}}^{2}+g_{\mathrm{V}}^{2}\right)$. If the $\tau$ rest frame is reconstructed, for example with the help of microvertex detectors, then $\vec{R} /\left(m_{\tau} \omega\right)$ can be evaluated in this frame. Since $R_{\mu} R^{\mu} /\left(m_{\tau} \omega\right)^{2}=-1$, the sensitivity is completely retained in this case.

\section{2. $H, A \rightarrow t \bar{t} \rightarrow\left(b W^{+}\right)+\left(\bar{b} W^{-}\right)$}

This partonic final state can be treated in direct analogy to the pionic two-particle $\tau$ decay. Including the non-zero mass effects, the angular distribution of the $W^{ \pm}$bosons is given by

$$
\begin{aligned}
& \frac{1}{\Gamma} \frac{d \Gamma\left(H, A \rightarrow W^{+} W^{-} b \bar{b}\right)}{d \cos \theta_{+}^{*} d \cos \theta_{-}^{*} d \phi^{*}} \\
& \quad=\frac{1}{8 \pi}\left[1+\frac{\left(m_{t}^{2}-2 m_{W}^{2}\right)^{2}}{\left(m_{t}^{2}+2 m_{W}^{2}\right)^{2}}\left[\cos \theta_{+}^{*} \cos \theta_{-}^{*} \mp \sin \theta_{+}^{*} \sin \theta_{-}^{*} \cos \phi^{*}\right]\right]
\end{aligned}
$$

$\theta_{ \pm}^{*}$ denote the $W^{ \pm}$polar angles, $\phi^{*}$ the azimuthal angle between the decay planes, Fig. 5 . The kinematical reconstruction will be much simpler in this case than for $\tau$ decays - despite the jetty character of the final state - as the mass ratio of daughter-to-parent particles is much larger and the decay polar angles are not driven to $0, \pi$ by Lorentz boosts. The angle $\phi^{*}$ is again determined only up to a two-fold ambiguity, but as before this ambiguity does not affect the distributions.

A particularly interesting process is provided by subsequent decays of the $W^{ \pm}$bosons to leptons. In this case too, the top quark direction can be reconstructed completely. The distribution obtained after integration over the $b$-quark directions is exactly the same as in eqn.(3) with the angles $\theta_{ \pm}^{*}$ denoting now the polar angles between the leptons and the top quarks in the quark rest frames. The form is similar to the distribution of the pions from the $\tau$ decay mode, and thus without the suppression factor $\left(\left(m_{t}^{2}-2 m_{W}^{2}\right) /\left(m_{t}^{2}+2 m_{W}^{2}\right)\right)^{2}$. Furthermore, the difference between scalar and pseudoscalar distributions is visible over a much larger angular range, as the Higgs-to-top boosts are generally small, see Fig. 6. 


\section{Higgs Decays to $W, Z$ Pairs}

Experimental opportunities to measure the parity of the Higgs particle in the decay processes

$$
H, A \rightarrow W^{+} W^{-} \text {and } Z Z
$$

have been investigated earlier [3, [4]. Nevertheless, for the sake of completeness, the main points are summarized here.

Even though the coupling of gauge bosons to the pseudoscalar state $A$ is not forbidden by any fundamental principle [as the decay $\pi^{0} \rightarrow \gamma \gamma$ tells us], the coupling vanishes at the Born level and proceeds only through triangular loop effects in two-doublet Higgs models [25]. However, independently of the low rate, this decay mode is useful theoretically by allowing us to check the uniqueness of the predictions for $0^{++}$decay distributions to the $W, Z$ bosons.

Vector particles couple to scalar Higgs particles in $\mathrm{S}$ waves, $\sim \epsilon_{1} \cdot \epsilon_{2}$, and they couple to pseudoscalar Higgs particles in $\mathrm{P}$ waves, $\sim\left(\vec{\epsilon}_{1} \times \vec{\epsilon}_{2}\right) \cdot \vec{k}_{V}$. While the transverse polarization vectors are independent of the energy, the longitudinal polarization vectors grow with the energy of the particle. As a result, the vector particles are polarized longitudinally for asymptotic energies in the $0^{++}$case, but they are polarized transversely, independently of the energy, in the $0^{-+}$case. If both vector bosons are real, the fraction of longitudinally polarized vector bosons is given by

$$
\frac{\Gamma_{L}(H, A \rightarrow V V)}{\Gamma_{L}+\Gamma_{T}}= \begin{cases}\frac{1-4 \rho+4 \rho^{2}}{1-4 \rho+12 \rho^{2}} & \text { for } H\left[0^{++}\right] \\ 0 & \text { for } A\left[0^{-+}\right]\end{cases}
$$

with $\rho=m_{V}^{2} / m_{H, A}^{2}$. The ratio grows from $1 / 3$ to 1 if the $0^{++}$Higgs mass rises from the $V V$ threshold to large values. The $V$ helicities can be determined by measuring the angular distributions of the decay products in $H, A \rightarrow V V \rightarrow\left(f_{1} \bar{f}_{2}\right)\left(f_{3} \bar{f}_{4}\right)$.

Defining two planes through the decays $V \rightarrow f_{1} \bar{f}_{2}$ and $V \rightarrow f_{3} \bar{f}_{4}$, the distribution of the azimuthal angle between the decay planes is given by [4]

$$
\frac{d \Gamma(H \rightarrow V V)}{d \phi^{*}} \sim 1+a_{1} \cos \phi^{*}+a_{2} \cos 2 \phi^{*}
$$

with

$$
\begin{aligned}
& a_{1}=-\frac{9 \pi^{2}}{32} \frac{\gamma_{1} \gamma_{3}\left(1+\beta_{1} \beta_{3}\right)}{\gamma_{1}^{2} \gamma_{3}^{2}\left(1+\beta_{1} \beta_{3}\right)^{2}+2} \frac{2 v_{1} a_{1}}{v_{1}^{2}+a_{1}^{2}} \frac{2 v_{3} a_{3}}{v_{3}^{2}+a_{3}^{2}} \\
& a_{2}=\frac{1}{2} \frac{1}{\gamma_{1}^{2} \gamma_{3}^{2}\left(1+\beta_{1} \beta_{3}\right)^{2}+2}
\end{aligned}
$$

for $0^{++}$particles and

$$
\frac{d \Gamma(A \rightarrow V V)}{d \phi^{*}} \sim 1-\frac{1}{4} \cos 2 \phi^{*}
$$


for $0^{-+}$particles. As expected the $\phi^{*}$ asymmetry vanishes for large scalar Higgs masses while it is independent of the pseudoscalar Higgs mass. For $V=W$, the charges are $v_{i}=a_{i}=1 ;$ for $V=Z, v_{i}=2 I_{3 i}-4 Q_{i} \sin ^{2} \theta_{W}$ and $a_{i}=2 I_{3 i} . \beta_{i}\left(\gamma_{i}\right)$ are the velocities $(\gamma$ factors) of the vector bosons. The angular distributions apply also to off-shell vector bosons below threshold; the fraction $L /(L+T)$ of longitudinally polarized vector bosons has been discussed for this case in Ref. 1 .

\section{Higgs-Strahlung in $e^{+} e^{-}$Collisions}

The analysis of the angular distributions in the Higgs-strahlung process

$$
e^{+} e^{-} \rightarrow Z H
$$

provides sensitive tests of the spin and parity of the scalar Higgs particle $H$ [4]. The explicit form of the angular distribution can be written

$$
\frac{d \sigma(Z H)}{d \cos \theta} \sim \beta^{2} \sin ^{2} \theta+8 \frac{m_{Z}^{2}}{s}
$$

For high energies the $Z$ boson is produced in a state of longitudinal polarization,

$$
\frac{\sigma\left(Z_{L}\right)}{\sigma\left(Z_{L}\right)+\sigma\left(Z_{T}\right)}=1-\frac{8}{12+\beta^{2} s / m_{Z}^{2}}
$$

with $\beta^{2}=\left[1-\left(m_{H}+m_{Z}\right)^{2} / s\right]\left[1-\left(m_{H}-m_{Z}\right)^{2} / s\right]$. By contrast, if the produced Higgs boson were a pseudoscalar particle, the $Z$ boson would be in a transversely polarized state, independently of the energy, and the angular distribution would read $d \sigma(Z A) / d \cos \theta=$ $1-\frac{1}{2} \sin ^{2} \theta$ instead. We thus conclude that the angular distribution and the fraction of Higgs-strahlung events with longitudinal $Z$ polarization provide tests which are sensitive to the spin and parity of the scalar Higgs particles.

\section{5. $\quad \gamma \gamma$ Production of Higgs Particles}

The colliding $\gamma$ beam reaction

$$
\gamma \gamma \rightarrow H, A
$$

has long been recognized (see e.g. [8 12]) as an important instrument to study the properties of Higgs particles. Since the $\gamma \gamma$ coupling, accessible through the cross section of this process

$$
\sigma(\gamma \gamma \rightarrow H, A)=\frac{8 \pi^{2}}{M} \Gamma(H, A \rightarrow \gamma \gamma) \frac{M \Gamma_{\mathrm{tot}} / \pi}{\left(s_{\gamma \gamma}-M^{2}\right)^{2}+\left(M \Gamma_{\mathrm{tot}}\right)^{2}}
$$

\footnotetext{
${ }^{2}$ The Breit-Wigner distribution can in general be approximated by the $\delta$-function $\delta\left(s_{\gamma \gamma}-M^{2}\right)$.
} 
is mediated by loops of all charged particles with non-zero mass, these measurements can virtually reveal the action of new particle species and help discriminate, for instance, the light Higgs boson of supersymmetric theories from that of the Standard Model. Using linearly polarized photon beams, the parity of the produced Higgs boson can be measured directly [12, 国. While the polarization vectors of the two photons must be parallel to generate $0^{++}$scalar particles, they must be perpendicular for $0^{-+}$pseudoscalar particles [14,

$$
\begin{aligned}
\mathcal{M}\left(\gamma \gamma \rightarrow H\left[0^{++}\right]\right) & \sim \vec{\epsilon}_{1} \cdot \vec{\epsilon}_{2} \\
\mathcal{M}\left(\gamma \gamma \rightarrow A\left[0^{-+}\right]\right) & \sim \vec{\epsilon}_{1} \times \vec{\epsilon}_{2} \cdot \vec{k}_{\gamma}
\end{aligned}
$$

High energy colliding beams of linearly polarized photons can be generated by Compton back-scattering of linearly polarized laser light on electron/positron bunches of $e^{+} e^{-}$linear colliders 13]. The linear polarization transfer from the laser photons to the high-energy photons is described by the $\xi_{3}$ component of the Stokes vector. The length of this vector depends on the final state photon energy and on the value of the parameter $x_{0}=4 E_{e} \omega_{0} / m_{e}^{2}$ where $E_{e}$ and $\omega_{0}$ are the electron/positron and laser energies, respectively. The requirement to operate below the $e^{ \pm}$pair threshold in collisions between the laser $\gamma$ 's and the high energy $\gamma$ 's implies the upper bound $x_{0} \lesssim 4.83$; lower bounds on $x_{0}$ depend on the available laser frequencies in the red/infrared regime. As shown in Fig. 7, the linear polarization transfer is large for small values of $x_{0}$ if the photon energy $y=E_{\gamma} / E_{e}$ is close to the maximum value, i.e.

$$
y \rightarrow y_{\max }=\frac{x_{0}}{1+x_{0}}
$$

for a given parameter $x_{0}$. Adopting the usual definition

$$
r=\frac{y}{x_{0}(1-y)} \leq 1
$$

[where the maximum is reached for $y \rightarrow y_{\max }$ ], the $\gamma$ energy spectrum is given by

$$
\phi_{0}(y)=\frac{1}{1-y}+1-y-4 r(1-r)
$$

and the Stokes component $\xi_{3}(y)=\phi_{3}(y) / \phi_{0}(y)$ follows from

$$
\phi_{3}(y)=2 r^{2}
$$

The maximum value of the Stokes vector $\xi_{3}(y)$ is reached for $y=y_{\max }$,

$$
\xi_{3}^{\max }=\frac{2\left(1+x_{0}\right)}{1+\left(1+x_{0}\right)^{2}}
$$

and approaches unity for small values of $x_{0}$.

The overall $\gamma \gamma$ luminosity is assumed to be of the same size as the $e^{+} e^{-}$collider luminosity. 
Since only part of the laser polarization is transferred to the high-energy photon beam, it is useful to define the polarization asymmetry $\mathcal{A}$ as

$$
\mathcal{A}=\frac{N^{\|}-N^{\perp}}{N^{\|}+N^{\perp}}
$$

where $N^{\|}$and $N^{\perp}$ denote the number of $\gamma \gamma$ events with the initial laser polarizations being parallel and perpendicular, respectively. It follows from eqn.(27) that

$$
\begin{aligned}
& \mathcal{A}\left(\gamma \gamma \rightarrow H\left[0^{++}\right]\right)=+\mathcal{A} \\
& \mathcal{A}\left(\gamma \gamma \rightarrow A\left[0^{-+}\right]\right)=-\mathcal{A}
\end{aligned}
$$

For resonance production, the asymmetry $\mathcal{A}$ can be expressed by the appropriate luminosity factors,

$$
\mathcal{A}=\frac{<\phi_{3} \phi_{3}>_{\tau}}{<\phi_{0} \phi_{0}>_{\tau}}
$$

with

$$
<\phi_{i} \phi_{i}>_{\tau}=\frac{1}{N^{2}} \int_{\tau / y_{\max }}^{y_{\max }} \frac{d y}{y} \phi_{i}(y) \phi_{i}(\tau / y)
$$

The Drell-Yan parameter is given by

$$
\tau=M^{2} / s_{e^{+} e^{-}}
$$

where $M$ is the resonance mass and $s_{e^{+} e^{-}}$is the $e^{ \pm}$collider energy-squared. The maximum value of $\tau$ is bounded by the energy transfer

$$
\begin{aligned}
\tau_{\max } & =y_{\max }^{2} \\
& =\left[\frac{x_{0}}{1+x_{0}}\right]^{2}
\end{aligned}
$$

The normalization factor $N$ is defined as

$$
\begin{aligned}
N & =\int_{0}^{y_{\max }} d y \phi_{0}(y) \\
& =\ln \left(1+x_{0}\right)\left[1-\frac{4}{x_{0}}-\frac{8}{x_{0}^{2}}\right]+\frac{1}{2}+\frac{8}{x_{0}}-\frac{1}{2\left(1+x_{0}\right)^{2}}
\end{aligned}
$$

The components $<\phi_{i} \phi_{i}>_{\tau}$ can easily be calculated, with the somewhat cumbersome results

$$
<\phi_{3} \phi_{3}>_{\tau}=\frac{1}{N^{2}} \frac{4 \tau^{2}}{x_{0}^{4}}\left\{2 \ln \left[\frac{\sqrt{\tau}}{x_{0}-\tau\left(1+x_{0}\right)}\right] \frac{\tau+1}{(\tau-1)^{3}}+\frac{2}{(\tau-1)^{2}} \frac{\tau\left(1+x_{0}\right)^{2}-x_{0}^{2}}{\tau-x_{0}+x_{0} \tau}\right\}
$$

and 


$$
\begin{aligned}
<\phi_{0} \phi_{0}>_{\tau}= & \frac{1}{N^{2}}\left\{\frac { 4 } { x _ { 0 } ^ { 4 } ( 1 + x _ { 0 } ) ( \tau - 1 ) ^ { 2 } } \frac { \tau ( 1 + x _ { 0 } ) ^ { 2 } - x _ { 0 } ^ { 2 } } { \tau - x _ { 0 } + x _ { 0 } \tau } \left[x_{0}^{3}(\tau-1)^{2}\left(\tau-x_{0}+x_{0} \tau\right)\right.\right. \\
& \left.-2 x_{0}\left(1+x_{0}\right)(\tau-1)\left(x_{0}\left(\tau^{2}-2 \tau+2\right)-4 \tau\right)+8 \tau^{2}\left(1+x_{0}\right)\right] \\
& +\ln \left[\frac{\sqrt{\tau}}{x_{0}-\tau\left(1+x_{0}\right)}\right] \frac{8}{x_{0}^{4}(\tau-1)^{2}}\left[2 x_{0}^{2}\left(2 \tau^{2}-4 \tau+1\right)+8 x_{0} \tau^{2}\right. \\
& \left.-x_{0}^{3}(\tau-1)\left(\tau^{2}-\tau+2\right)+\frac{x_{0}^{4}}{4}(\tau-1)\left(\tau^{2}-2 \tau+2\right)+\frac{4 \tau^{2}}{(\tau-1)}(\tau+1)\right] \\
& \left.+\ln \left[\frac{x_{0}}{\sqrt{\tau}\left(1+x_{0}\right)}\right] \frac{4}{x_{0}^{2}}\left[2 x_{0}(\tau+2)+x_{0}^{2}+4\right]\right\}
\end{aligned}
$$

The correlation functions $<\phi_{i} \phi_{i}>_{\tau}$ and the asymmetry $\mathcal{A}$ are displayed in Figs. 8 and 9. As expected, the maximum sensitivity

$$
\begin{aligned}
\mathcal{A}_{\max } & =\left(\xi_{3}^{\max }\right)^{2} \\
& =\left[\frac{2\left(1+x_{0}\right)}{1+\left(1+x_{0}\right)^{2}}\right]^{2}
\end{aligned}
$$

is reached for small values of $x_{0}$ and near the upper bound of $\tau \lesssim \tau_{\max }\left(x_{0}\right)$, i. e. if the energy is just sufficient to produce the Higgs particles in the $\gamma \gamma$ collisions. [5 Typical energies for electron/positron and laser beams are shown in Table 1 for a sample of $x_{0}$ values corresponding to large and small asymmetries $\mathcal{A}_{\max }$.

The measurement of the Higgs parity in $\gamma \gamma$ collisions will be a unique method in areas of the parameter space where the Higgs coupling to heavy $W, Z$ bosons are small and the top quark decay channels are closed so that the Higgs particles decay preferentially to $b, c, \tau$ fermions. This method is therefore most useful for particles in extended Higgs models while the profile of the $\mathcal{S M}$ Higgs particle can be determined in Higgs decay and $e^{+} e^{-}$production processes.

It must therefore be shown that the background events from heavy quark production, $\gamma \gamma \rightarrow b \bar{b}$ and $c \bar{c}$, can be suppressed sufficiently well. This is a difficult task [8, 15] for $b$ quarks. For charm quarks the situation looks superficially even more miserable since the $c \bar{c}$ production cross sections are $\sim 16 / 4 / 1$ times larger than the $b \bar{b}$ cross sections for direct/1resolved $/ 2-$-resolved $\gamma$ events at high energies. However, to simplify the discussion we shall assume, for the time being, $\mu$-vertexing to be perfect and we neglect the contamination from $c \bar{c}$ production. [This is actually not far from reality, as discussed in Ref. 11.]

Three components contribute to the $b \bar{b}$ background events: direct $\gamma \gamma$ production, the $1-$ resolved photon process $\gamma \gamma(\rightarrow g) \rightarrow b \bar{b}$, and the 2 -resolved photon process $\gamma \gamma(\rightarrow g g) \rightarrow b \bar{b}$ 8.15,26.

\footnotetext{
${ }^{3}$ Since the luminosity vanishes at $\tau=\tau_{\max }$, one will set the operating conditions in practice such that $\tau \lesssim \tau_{\max }$ allows for a sufficiently large luminosity $<\phi_{0} \phi_{0}>_{\tau}$, as illustrated in Figs. 10 to 13.
} 


\begin{tabular}{|c|cc|c|cc|}
\hline$x_{0}$ & $\mathcal{A}_{\max }$ & $\sqrt{\tau_{\max }}$ & $M_{H}[\mathrm{GeV}]$ & $\begin{array}{c}E_{e}[\mathrm{GeV}] \\
\left(\mathrm{at} \sqrt{\tau_{\max }}\right)\end{array}$ & $\omega_{0}[\mathrm{eV}]$ \\
\hline \hline 0.5 & 0.85 & 0.33 & 100 & 150 & 0.22 \\
& & & 200 & 300 & 0.11 \\
& & & 300 & 450 & 0.07 \\
\hline 1.0 & 0.64 & 0.5 & 100 & 100 & 0.65 \\
& & & 200 & 200 & 0.33 \\
& & & 300 & 300 & 0.22 \\
\hline 2.0 & 0.36 & 0.67 & 100 & 75 & 1.74 \\
& & & 200 & 150 & 0.87 \\
& & & 300 & 225 & 0.58 \\
\hline 4.83 & 0.11 & 0.83 & 100 & 60.4 & 5.22 \\
& & & 200 & 121 & 2.61 \\
& & & 300 & 181 & 1.74 \\
\hline
\end{tabular}

Table 1: Electron/positron energies $E_{e}$ of the $e^{+} e^{-}$linear collider and laser $\gamma$ energies $\omega_{0}$ for a sample of Higgs masses if the parameter $x_{0}=4 E_{e} \omega_{0} / m_{e}^{2}$, which determines the maximum asymmetry $\mathcal{A}_{\max }$ at $\sqrt{\tau_{\max }}=y_{\max }$, is varied from small to large values.

The cross section for direct $\gamma \gamma$ production

$$
\gamma \gamma \rightarrow b \bar{b}
$$

can be easily calculated at the tree level for linearly-polarized photons. Effects due to higher-order QCD corrections have been shown to be modest in the unpolarized case [26] and, hence, can be safely neglected for asymmetries. The cross sections are given by

$$
\begin{aligned}
\frac{d \sigma^{\|}}{d y d \phi} & =\frac{6 \alpha^{2} Q_{b}^{4}}{s} \frac{1+e^{-4 y}+2 e^{-2 y} \sin 4 \phi}{\left(1+e^{-2 y}\right)^{2}} \\
\frac{d \sigma^{\perp}}{d y d \phi} & =\frac{6 \alpha^{2} Q_{b}^{4}}{s} \frac{1+e^{-4 y}-2 e^{-2 y} \sin 4 \phi}{\left(1+e^{-2 y}\right)^{2}}
\end{aligned}
$$

for parallel and perpendicular photon polarizations, respectively, at energies sufficiently above the quark threshold. Note that $y$ denotes here the rapidity of the $b$ quark and $\phi$ the azimuthal angle of the production plane with respect to either of the photon polarization vectors in the center-of-mass frame. If only one of the photon beams is linearly polarized and the second beam unpolarized, the cross section does not depend on the azimuthal angle $\phi$ for energies sufficiently above the $b \bar{b}$ threshold. The unpolarized cross section is the average of the parallel and perpendicular cross sections. As evident from eqs.(36), the background process $\gamma \gamma \rightarrow b \bar{b}$ does not affect the numerator of the asymmetry $\mathcal{A}$ if the azimuthal angle 
is integrated over, yet it does increase the denominator, i.e. $N^{\|}+N^{\perp} \rightarrow\left[N^{\|}+N^{\perp}\right]_{\text {Res }}+$ $\left[N^{\|}+N^{\perp}\right]_{\mathrm{bkgd}}$, thus diluting the asymmetry in general by a significant amount.

If one or two photons are resolved into quark-plus-gluon showers, the subprocesses $\gamma g \rightarrow$ $b \bar{b}$ and $g g \rightarrow b \bar{b}$ generate $b$-quark final states which are accompanied by hadron jets spraying into in the photon direction(s). Since gluons are generated only in the double-splitting process $\gamma \rightarrow q \rightarrow g$, the gluon spectrum in the photon falls off steeply with the gluon momentum. Therefore, the 1-and 2-resolved photon processes are strongly suppressed if nearly all the photon energy is needed to generate the $b \bar{b}$ final state energy. This is however the situation we encounter when the asymmetry $\mathcal{A}$ in Higgs production will be measured for $\tau$ values close to $\tau_{\max }\left(x_{0}\right)$, cf. Fig. 9. The background from 1-resolved processes is therefore expected to be small in this kinematical configuration and negligible for the 2resolved photon processes. [A detailed example will be presented in Fig. 10(a).] The resolved photon mechanisms therefore do not pose background problems in our case.

While the signal events $\gamma \gamma \rightarrow H, A \rightarrow b \bar{b}$ are distributed isotropically in their centerof-mass frame, the background events are strongly peaked at zero polar angles. This can be exploited to reject the background events by demanding small values $\left(\left|y_{b, \bar{b}}\right| \lesssim 1\right)$ for the rapidities of the $b$ and $\bar{b}$ quarks. In Figs. 10 to $13(\mathrm{a})$ we compare the (unpolarized) signal cross sections for $H_{S M}$ in the Standard Model and $h^{0} / H^{0} / A^{0}$ in the minimal supersymmetric model at $\tan \beta=1.5$ and 30 with the background $b \bar{b}$ channels integrated over rapidities $\left|y_{b, \bar{b}}\right| \leq 1$. We have assumed a mass resolution $\Delta M=5 \mathrm{GeV}$. examples $x_{0}$ has been chosen $=1$ and the $\tau$ values have been set slightly below $\tau_{\text {max }}$ to allow for sufficiently large luminosities at the resonance $\gamma \gamma$ energy. It is clear from these figures that the measurement of the Higgs parity, in particular for the heavy particles, requires high $\gamma \gamma$ luminosities. The background events reduce the asymmetry $\mathcal{A}$ by a suppression factor $1 /[1+B / S]$ where $S(B)$ denote the number of signal (background) events. The asymmetries including background events are displayed in Figs. 10 to 13(b) for the Standard Model Higgs particle $H_{S M}$ and for the $h^{0} / H^{0} / A^{0}$ particles in the minimal supersymmetric model for $\tau$ values slightly below $\tau_{\max }$ where the polarization asymmetry is maximal. If an integrated luminosity of $\int \mathcal{L}=100 \mathrm{fb}^{-1}$ can be accumulated within a few years, asymmetries down to $\sim 3 \%$ will be accessible, covering major parts of the intermediate Higgs mass range in the Standard Model and of the supersymmetric parameter space. To carry out these experiments, laser operations in the red/infrared regime are necessary in order to generate sufficiently high asymmetries.

Under these conditions the polarization asymmetry of the $\mathcal{S M}$ Higgs particle $H_{S M}$ can be measured in $\gamma \gamma$ collisions throughout the relevant mass range below $\sim 150 \mathrm{GeV}$ in the $b \bar{b}$ channel; above this mass value Higgs decays to $Z$ bosons (virtual/real) can be exploited to determine spin and parity. The light scalar $\mathcal{M S S M}$ Higgs boson $h^{0}$ can be probed in a similarly comprehensive way, except presumably for the low mass range at large $\tan \beta$. By contrast, the measurement of the polarization asymmetry of the heavy scalar $\mathcal{M S S M}$ Higgs particle $H^{0}$ appears to be very difficult in the $b \bar{b}$ channel. In this

\footnotetext{
${ }^{4}$ Coulomb-gluon exchange between the top-antitop quarks mediating the $\gamma \gamma$ coupling to the $A^{0}$ Higgs boson, leads to pseudoscalar S-wave $[t \bar{t}]$ resonance contributions near the threshold, and the lowest-order analysis cannot be applied any more in this range, Ref. 27.
} 
case decays to gauge bosons and top quark pairs, as well as the angular distribution in the Higgs-strahlung production process are more promising, as evident from Fig. 1. Finally, the $\gamma \gamma$ polarization measurement of the parity in the very interesting case of the pseudoscalar $A^{0}$ Higgs particle appears feasible throughout most of the parameter range below the top threshold; $A^{0} \rightarrow t \bar{t}$ decays can be exploited for masses above this threshold.

These figures represent illustrative examples. Optimizing the run parameters $\left[x_{0} / y / \Delta y_{b \bar{b}}\right]$ will help refine the analysis if necessary.

\section{Conclusions}

The analyses in the preceding sections provide a transparent picture of prospects to determine the external quantum numbers $J^{P C}=0^{++}$and $0^{-+}$of the scalar and pseudoscalar Higgs particles experimentally.

(i) For scalar $0^{++}$Higgs particles the measurement is easy if the particles couple strongly enough to the $W, Z$ gauge bosons. In this case Higgs decays to vector boson pairs can be exploited at the LHC and $e^{+} e^{-}$linear colliders if the Higgs mass is sufficiently large. In the intermediate mass range, the production through Higgs-strahlung off the (virtual/real) $Z$ boson in $e^{+} e^{-}$collisions provides a complementary method. The angular distributions of the decay and production processes are characteristic for the spin and parity of the Higgs particles. This has been demonstrated by confronting the $0^{++}$predictions with distributions derived for other assignments of these quantum numbers. While the $\mathcal{S} \mathcal{M}$ Higgs sector can be covered completely this way, the method can be applied only in a restricted range of the $\mathcal{S U S Y}$ Higgs parameter space.

(ii) Since the pseudoscalar $A^{0}$ Higgs boson in supersymmetric extensions of the Standard Model does not couple directly to gauge bosons, other methods must be used to measure its parity. Above the $t \bar{t}$ decay threshold, correlations among the $(b W)$ decay products of the $t$ and $\bar{t}$ quarks allow us to determine the negative parity in a straightforward way. Analogous information is provided by the hadronic decays of $\tau$-pairs produced in Higgs decays below the top pair production threshold.

(iii) In major areas of the $\mathcal{S U S \mathcal { Y }}$ parameter space the fusion of Higgs particles in linearly polarized $\gamma \gamma$ collisions provides an alternative method to these classical tools. Parallel / perpendicular $\gamma \gamma$ beams generate $0^{++} / 0^{-+}$Higgs particles, respectively. By choosing the frequency of the laser light in the Compton back-scattering process for generating high energy $\gamma$ beams as low as possible and the original $e^{ \pm}$energy just high enough to produce the Higgs particles in the $\gamma \gamma$ collisions, one can find an optimum point for operating the experiment at which the asymmetry is maximal and the Higgs production rate sufficiently large. This is a particularly interesting method for measuring the negative parity of $A^{0}$ Higgs bosons in $\mathcal{S U S Y}$ extensions of the Standard Model which is hard to determine otherwise. In summa Besides the measurement of the Higgs $\gamma \gamma$ width, the prospects of determining the external quantum numbers of the Higgs particles render dedicated $\gamma \gamma$ collisions a very interesting instrumental option for future $e^{+} e^{-}$linear colliders. 


\section{Acknowledgements}

The authors would like to thank K. Hagiwara for helpful discussions. This work is supported in part by BMFT under contract 056KA93P, by KBN under grant 2P30225206 and by Deutsche Forschungsgemeinschaft DFG. MK gratefully acknowledges the hospitality extended to him by the DESY Theory Group. 


\section{References}

[1] P. W. Higgs, Phys. Rev. Lett. 12 (1964) 132 and Phys. Rev. 145 (1966) 1156;

F. Englert and R. Brout, Phys. Rev. Lett. 13 (1964) 321;

G. S. Guralnik, C. R. Hagen and T. Kibble, Phys. Rev. Lett. 13 (1964) 585.

[2] J. F. Gunion and H. E. Haber, Nucl. Phys. B272 (1986) 1 and B278 (1986) 449.

[3] J. R. Dell' Aquila and C. A. Nelson, Nucl. Phys. B320 (1989) 61.

[4] V. Barger, K. Cheung, A. Djouadi, B. A. Kniehl and P. M. Zerwas, Phys. Rev. D49 (1994) 79 .

[5] K. Hagiwara and M. L. Stong, Preprint MAD-PH-734 (Z. Phys. C, to appear).

[6] B. K. Bullock, K. Hagiwara and A. D. Martin, Phys. Rev. Lett. 67 (1991) 3055 and Phys. Lett. B273 (1991) 501.

[7] J. R. Dell' Aquila and C. A. Nelson, Nucl. Phys. B320 (1989) 86.

[8] J. F. Gunion and H. E. Haber, Phys. Rev. D48 (1993) 5109.

[9] P. M. Zerwas, Proceedings of the VIII Int. Workshop on Photon-Photon Collisions, Shoresh (Jerusalem Hills) 1988.

[10] S. J. Brodsky, Proceedings, II. Workshop on "Physics and Experiments with Linear $e^{+} e^{-}$Colliders", Waikoloa, 1993;

J. F. Gunion, ibid.

[11] D. L. Borden, D. A. Bauer, D. O. Caldwell, SLAC-PUB-5715 and Phys. Rev. D48 (1993) 4018.

[12] B. Grzadkowski and J. F. Gunion, Phys. Lett. B294 (1992) 361.

[13] I. F. Ginzburg, G. L. Kotkin, S. L. Panfil, V. G. Serbo, and V. I. Telnov, Nucl. Instr. and Meth. 219 (1984) 5.

[14] C. N. Yang, Phys. Rev. 77 (1949) 242.

[15] O. J. P. Eboli, M. C. Gonzales-Garcia, F. Halzen and D. Zeppenfeld, Phys. Rev. D48 (1993) 1430.

[16] G. V. Jikia, Phys. Lett. B298 (1993) 224.

[17] A. Djouadi, D. Haidt and P. M. Zerwas, Proceedings, " $e^{+} e^{-}$Colliders at $500 \mathrm{GeV}$ : The Physics Potential", DESY 92-123A;

R. Kleiss, Z. Kunszt and W. J. Stirling, Phys. Lett. B253 (1991) 269.

[18] B. A. Kniehl, Report DESY 93-069.

[19] A. Djouadi, J. Kalinowski and P. M. Zerwas, Proceedings, " $e^{+} e^{-}$Colliders at $500 \mathrm{GeV}$ : The Physics Potential", and Z. Phys. C57 (1993) 569. 
[20] B. Mele and G. Altarelli, Phys. Lett. B299 (1993) 345;

B. Mele, Proceedings, XXVII Recontres de Moriond, Les Arcs 1992 and Preprint Rome-1009-1994.

[21] K. Hagiwara, H. Murayama and I. Watanabe, Nucl. Phys. 367 (1991) 257;

H. Veltman, Preprint SACLAY-SPHT-93-111.

[22] J. H. Kühn, Acta Phys. Aus. Suppl. 24 (1982) 203;

I. I. Y. Bigi, Y. L. Dokshitser, V. Khoze, J. H. Kühn and P. M. Zerwas, Phys. Lett. 181B (1986) 157.

[23] J. H. Kühn and F. Wagner, Nucl. Phys. B236 (1984) 16.

[24] J. H. Kühn, Phys. Lett. B13 (1993) 458.

[25] A. Méndez, Proceedings, " $e^{+} e^{-}$Colliders at $500 \mathrm{GeV}$ : The Physics Potential", DESY 92-123A.

[26] M. Drees, M. Krämer, J. Zunft and P. M. Zerwas, Phys. Lett. B306 (1993) 371;

J. H. Kühn, E. Mirkes and J. Steegborn, Z. Phys. C57 (1993) 615.

[27] A. Djouadi, M. Spira, and P. M. Zerwas, Phys. Lett. B311 (1993) 255. 


\section{Figures}

Fig. 1. Branching ratios of Higgs decay modes in the Standard Model and its minimal supersymmetric extension. For the sake of clarity only those branching ratios are displayed which are of interest for the subsequent analyses. The top mass has been set to $m_{t}=150 \mathrm{GeV}$.

Fig. 2. Definition of the pion angles $\theta_{ \pm}^{*}$ and $\phi^{*}$ in the decays $\tau^{ \pm} \rightarrow \pi^{ \pm} \stackrel{(-)}{\nu_{\tau}}$. The polar angles $\theta_{ \pm}^{*}$ are defined in the $\tau_{ \pm}$rest frames, with respect to the $\tau^{-}$direction. $\phi^{*}$ remains unchanged under boosts along the $\tau^{+}, \tau^{-}$axis. The angle $\delta$ separates the pions in the Higgs rest frame; it is close to $\pi$ because of the large Lorentz boosts from $\tau$ to Higgs rest frames.

Fig. 3. Distributions of the decay $H, A \rightarrow \tau^{+} \tau^{-} \rightarrow \pi^{+} \bar{\nu}_{\tau} \pi^{-} \nu_{\tau}$ in the angle between the pions as measured in the Higgs rest frame. The plot is for non-zero pion mass and for $m_{H, A}=60,150 \mathrm{GeV}$. The distributions for scalar (pseudoscalar) Higgs particles are drawn with solid (dashed) lines.

Fig. 4. Distributions for $H, A \rightarrow \tau^{+} \tau^{-} \rightarrow \rho^{+} \bar{\nu}_{\tau} \rho^{-} \nu_{\tau}$ in the angle between the $\rho$ 's. The difference between scalar (solid) and pseudoscalar (dashed) distributions is much smaller than in the pion case. This effect is due mainly to the suppression factor $\left(m_{\tau}^{2}-2 m_{\rho}^{2}\right)^{2} /\left(m_{\tau}^{2}+2 m_{\rho}^{2}\right)^{2}$ discussed in the text.

Fig. 5. Definitions of the angles $\theta_{ \pm}^{*}$ and $\phi^{*}$ in the decay $H \rightarrow t \bar{t} \rightarrow\left(b W^{+}\right)+\left(\bar{b} W^{-}\right)$. The polar angles $\theta_{ \pm}^{*}$ are defined in the $t$ and $\bar{t}$ rest frames, with respect to the $t$ flight direction. The azimuthal angle $\phi^{*}$ remains unchanged under boosts along the $t, \bar{t}$ axis.

Fig. 6. Distributions of the decays $H, A \rightarrow t \bar{t} \rightarrow\left(b l^{+} \nu\right)\left(\bar{b} l^{-} \bar{\nu}\right)$ in the angle between the charged leptons. For massless $b$ 's and leptons, the only remaining dependence is on the ratio of top to Higgs masses. Scalar (solid) and pseudoscalar (dashed) distributions are shown for $m_{t}=150 \mathrm{GeV}$ and $m_{H, A}=400,1000 \mathrm{GeV}$.

Fig. 7. The Stokes component $\xi_{3}(y)$, determining the high energy $\gamma$ polarization, for various values of $x_{0}=4 E_{e} \omega_{0} / m_{e}^{2}\left[E_{e}=e^{ \pm}\right.$colliding beam energy; $\omega_{0}=$ laser energy].

Fig. 8. The luminosity components $<\phi_{3} \phi_{3}>_{\tau}$ and $<\phi_{0} \phi_{0}>_{\tau}$ building up the linear polarization asymmetry $\mathcal{A}$.

Fig. 9. The polarization asymmetry $\mathcal{A}$ for various values of $x_{0}$.

Fig. 10. Standard Model Higgs particle $H_{S M}$ : signal and background cross sections for $b \bar{b}$ final states (a), and the polarization asymmetry $\mathcal{A}$ including the background process (b). The top mass has been chosen $m_{t}=150 \mathrm{GeV}$ in this and the subsequent figures.

Fig. 11. $\mathcal{M S S M}$ Higgs particle $h^{0}$ : signal and background cross sections for $b \bar{b}$ final states (a), and the polarization asymmetry $\mathcal{A}$ including the background process (b). 
Fig. 12. $\mathcal{M S S M}$ Higgs particle $H^{0}$ : signal and background cross sections for $b \bar{b}$ final states (a), and the polarization asymmetry $\mathcal{A}$ including the background process (b).

Fig. 13. $\mathcal{M S} \mathcal{S} M$ Higgs particle $A^{0}$ : signal and background cross sections for $b \bar{b}$ final states (a), and the polarization asymmetry $\mathcal{A}$ including the background process (b). 
This figure "fig1-1.png" is available in "png" format from: http://arxiv.org/ps/hep-ph/9404280v1 

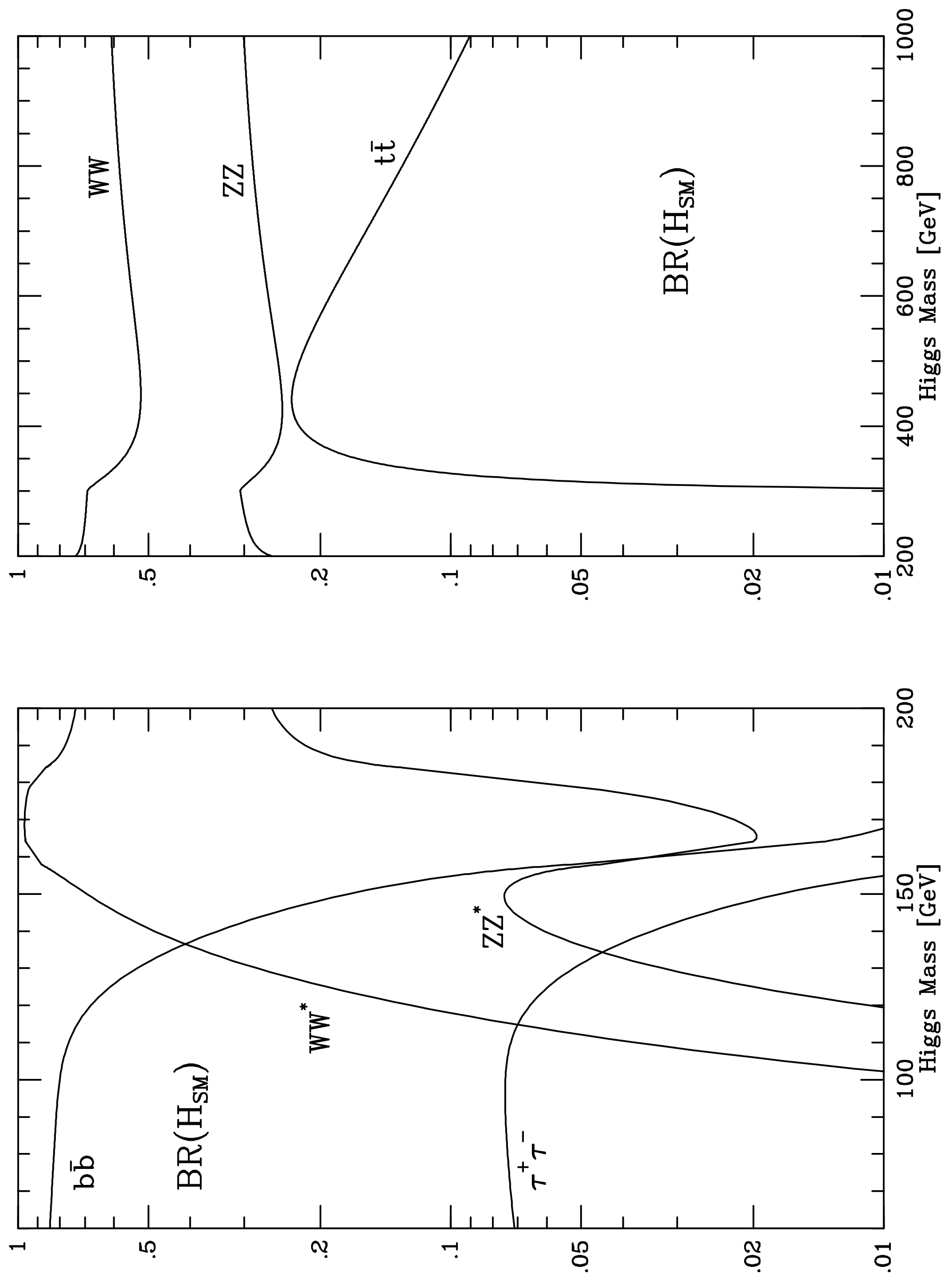


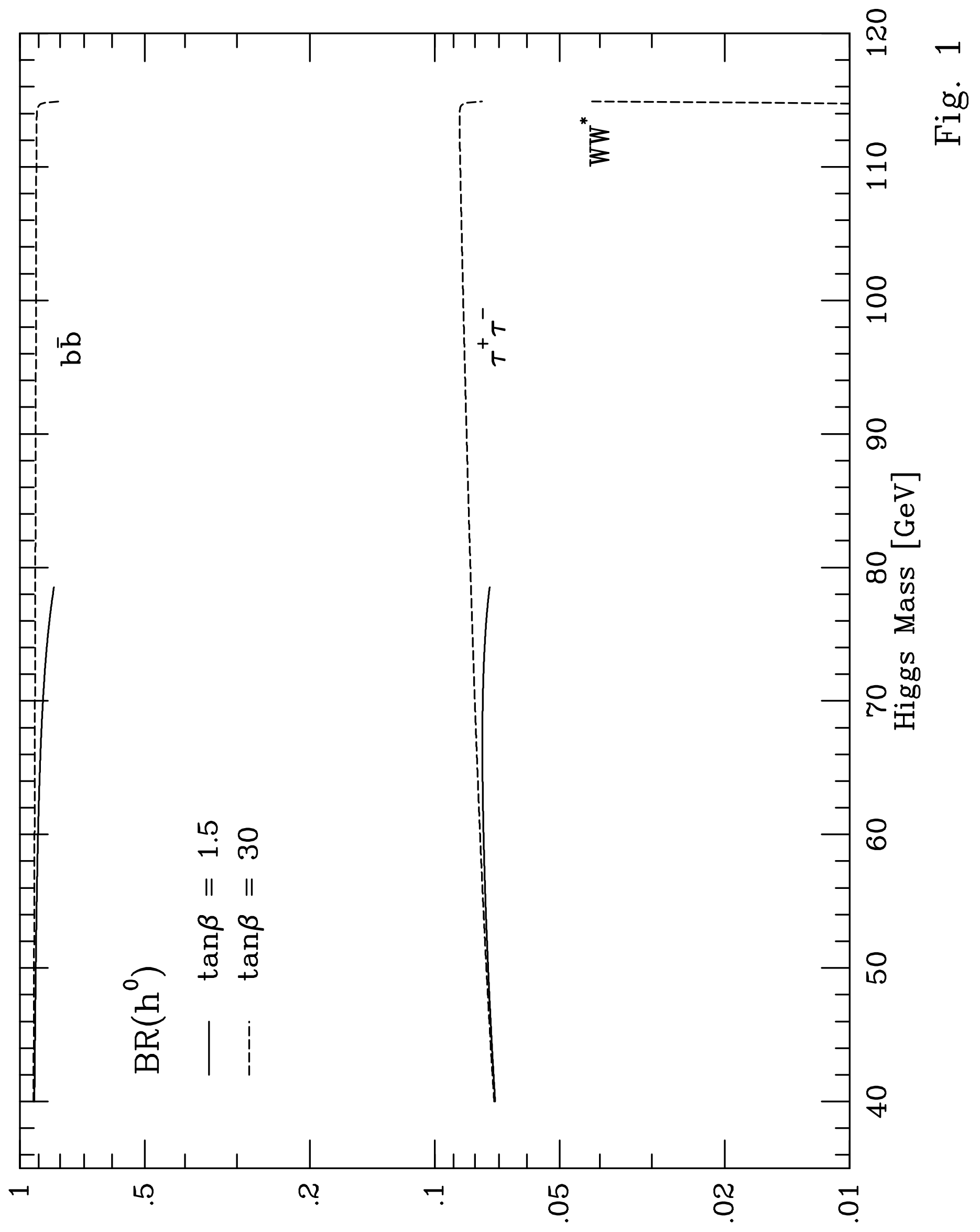



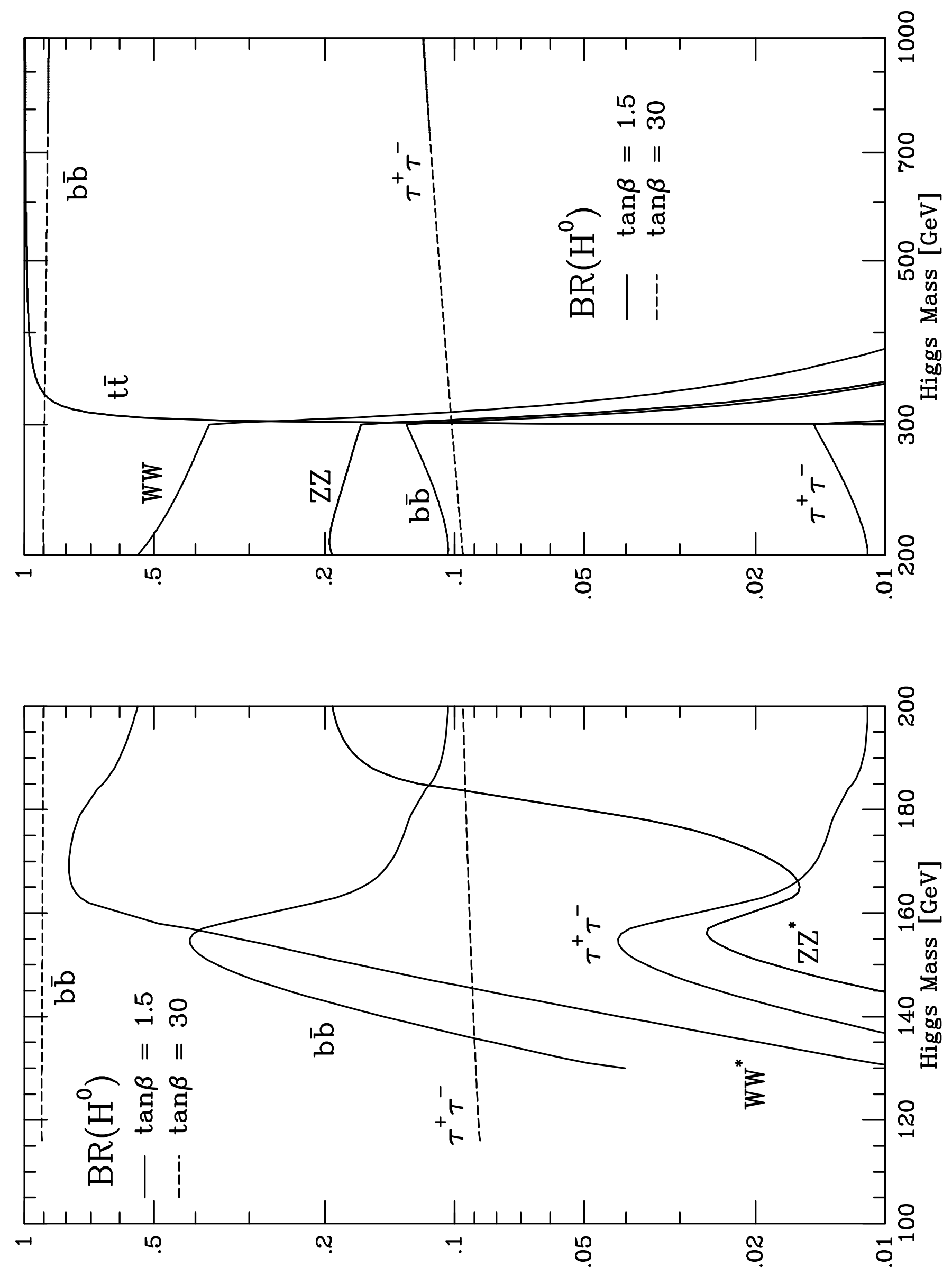


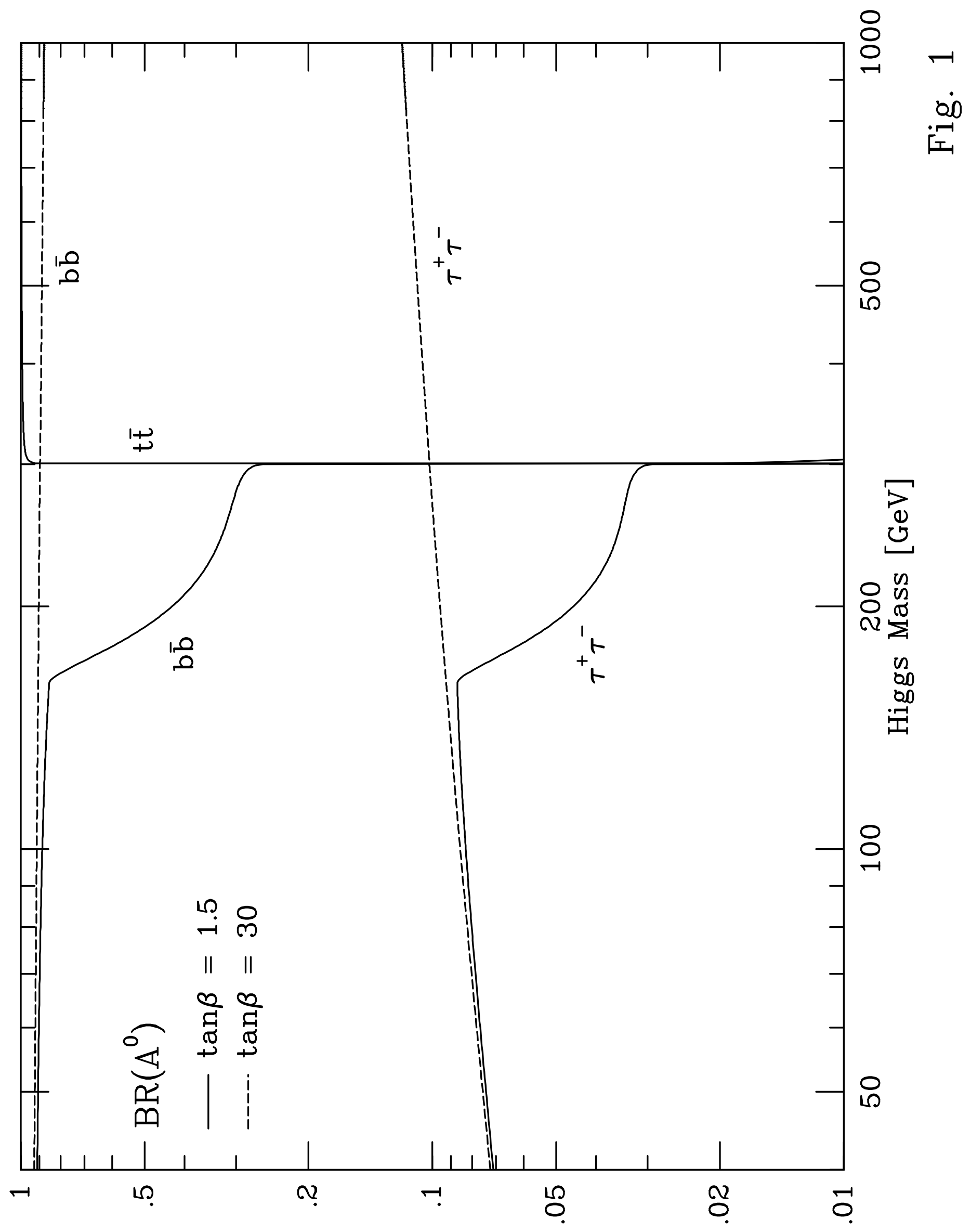


This figure "fig2-1.png" is available in "png" format from: http://arxiv.org/ps/hep-ph/9404280v1 
This figure "fig3-1.png" is available in "png" format from: http://arxiv.org/ps/hep-ph/9404280v1 
This figure "fig1-2.png" is available in "png" format from: http://arxiv.org/ps/hep-ph/9404280v1 
This figure "fig2-2.png" is available in "png" format from: http://arxiv.org/ps/hep-ph/9404280v1 

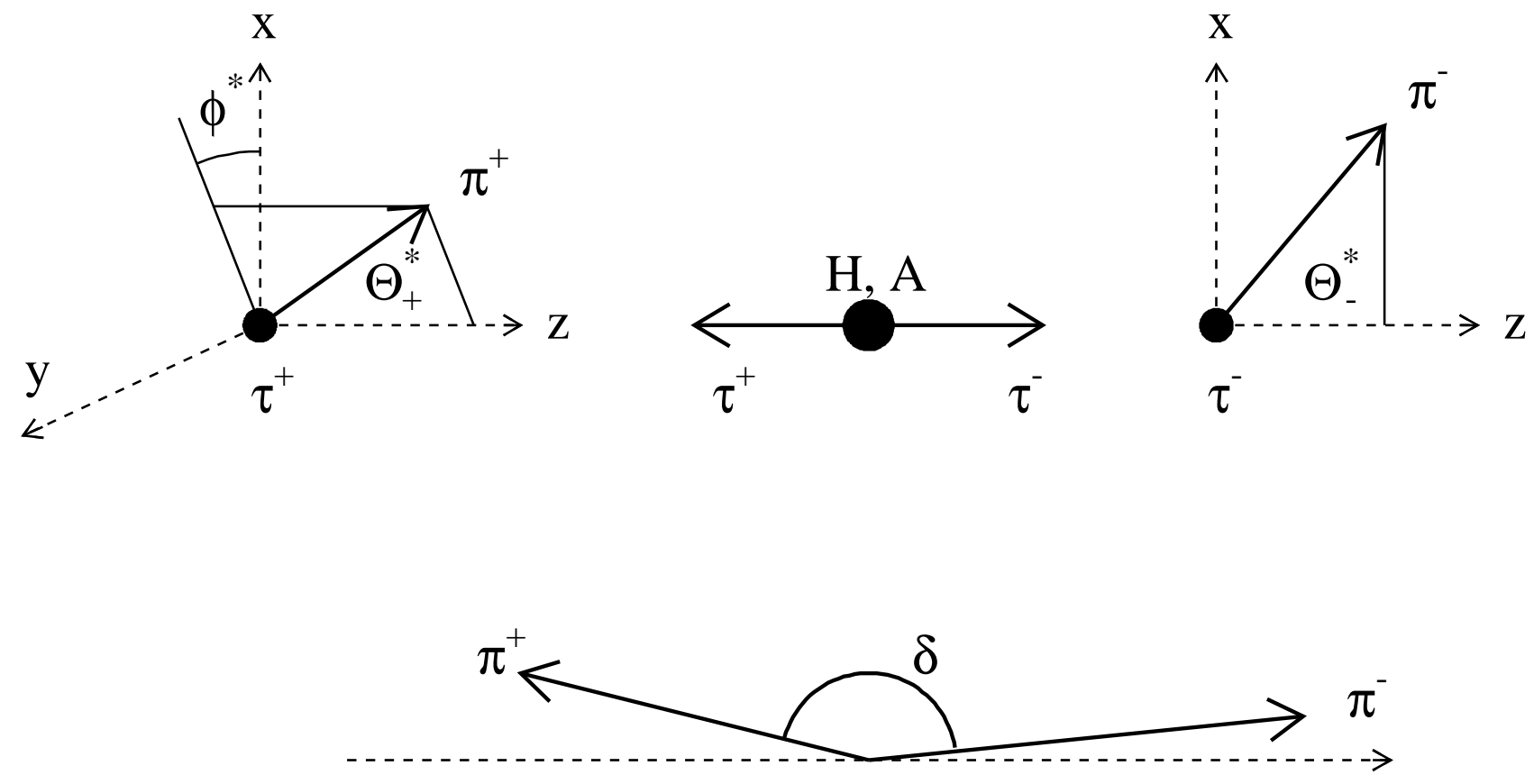
This figure "fig3-2.png" is available in "png" format from: http://arxiv.org/ps/hep-ph/9404280v1 
This figure "fig1-3.png" is available in "png" format from: http://arxiv.org/ps/hep-ph/9404280v1 
This figure "fig2-3.png" is available in "png" format from: http://arxiv.org/ps/hep-ph/9404280v1 
This figure "fig3-3.png" is available in "png" format from: http://arxiv.org/ps/hep-ph/9404280v1 


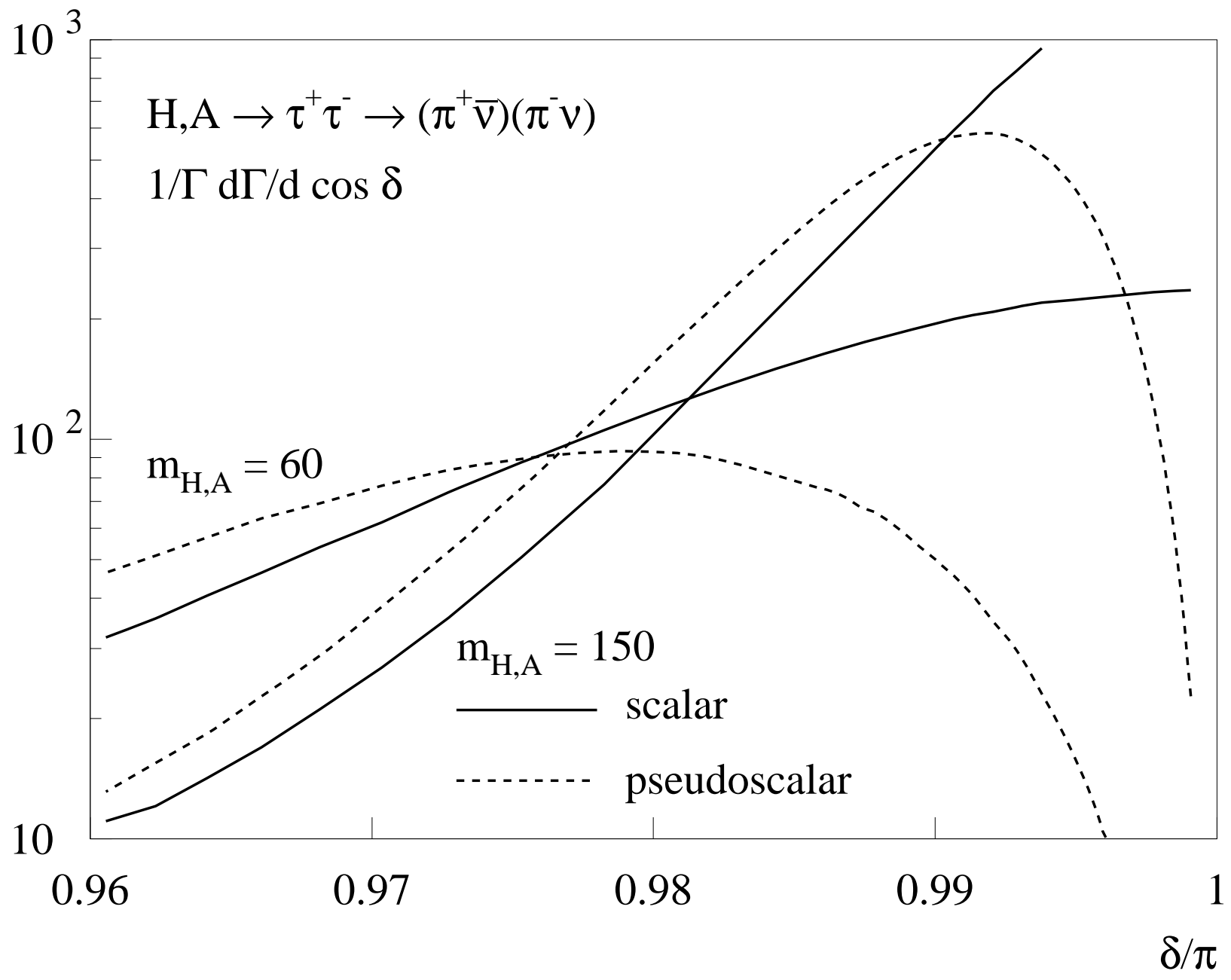


This figure "fig1-4.png" is available in "png" format from: http://arxiv.org/ps/hep-ph/9404280v1 
This figure "fig2-4.png" is available in "png" format from: http://arxiv.org/ps/hep-ph/9404280v1 
This figure "fig3-4.png" is available in "png" format from: http://arxiv.org/ps/hep-ph/9404280v1 


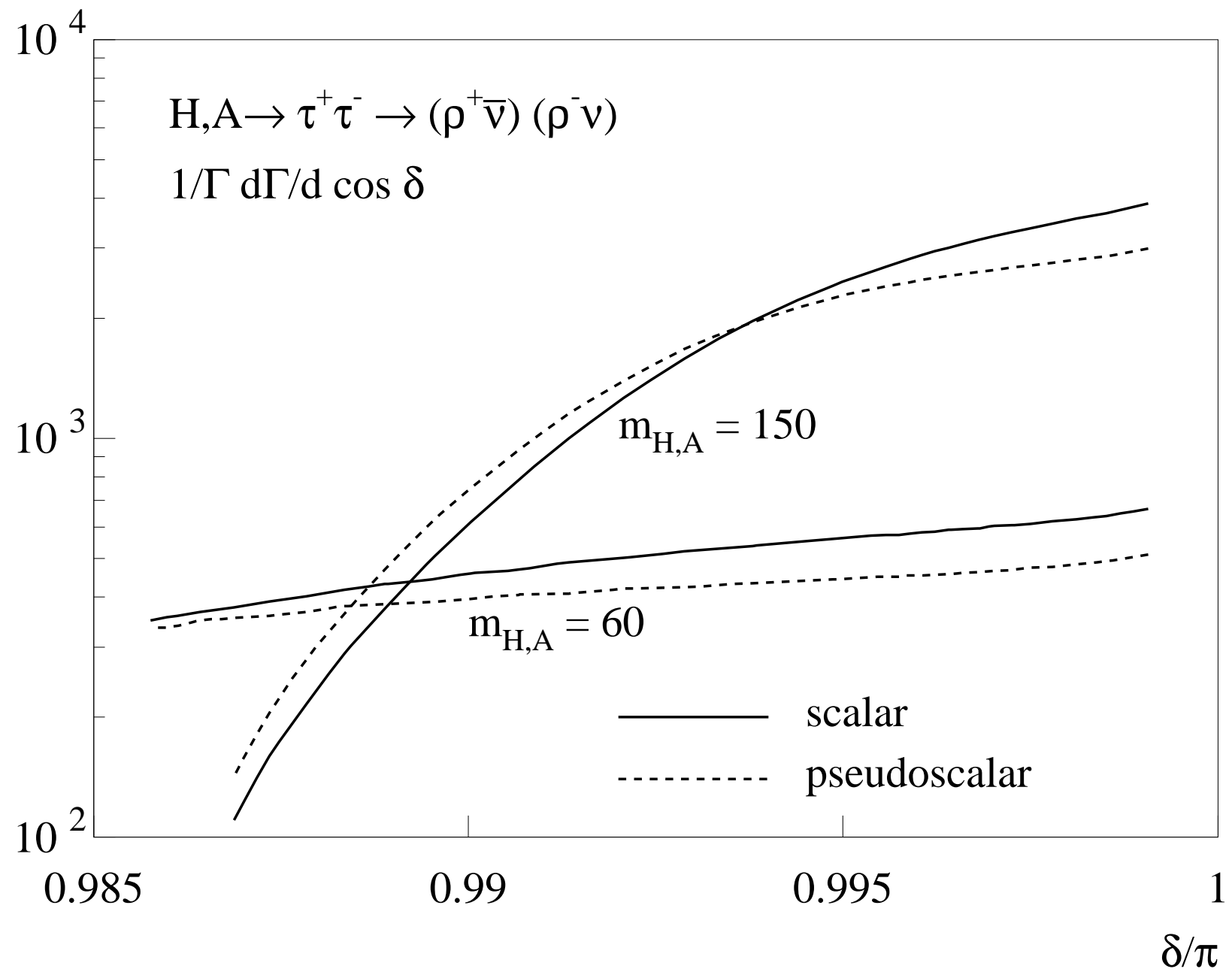


This figure "fig1-5.png" is available in "png" format from: http://arxiv.org/ps/hep-ph/9404280v1 
This figure "fig2-5.png" is available in "png" format from: http://arxiv.org/ps/hep-ph/9404280v1 
This figure "fig3-5.png" is available in "png" format from: http://arxiv.org/ps/hep-ph/9404280v1 
This figure "fig1-6.png" is available in "png" format from: http://arxiv.org/ps/hep-ph/9404280v1 
This figure "fig2-6.png" is available in "png" format from: http://arxiv.org/ps/hep-ph/9404280v1 
This figure "fig3-6.png" is available in "png" format from: http://arxiv.org/ps/hep-ph/9404280v1 


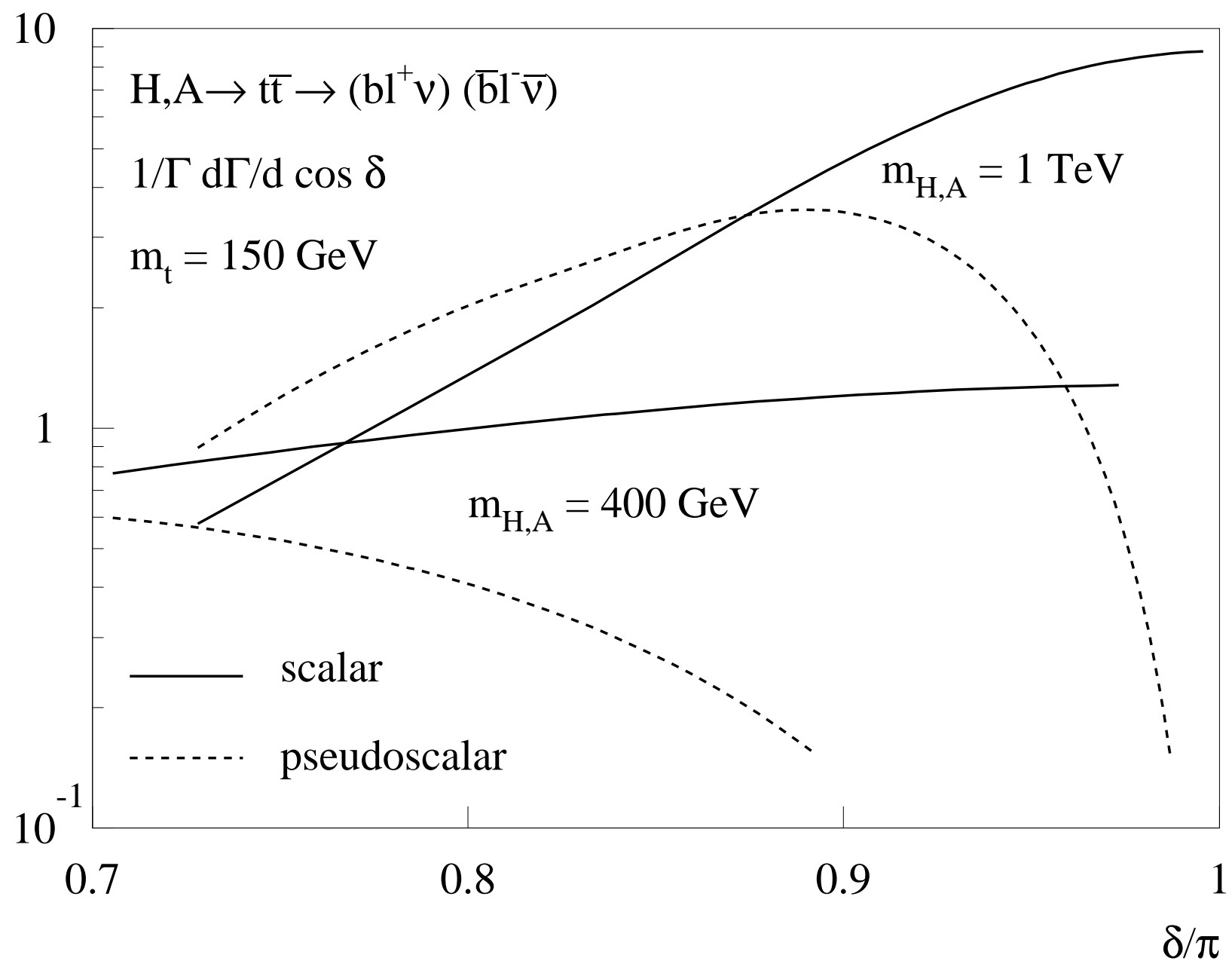


This figure "fig3-7.png" is available in "png" format from: http://arxiv.org/ps/hep-ph/9404280v1 


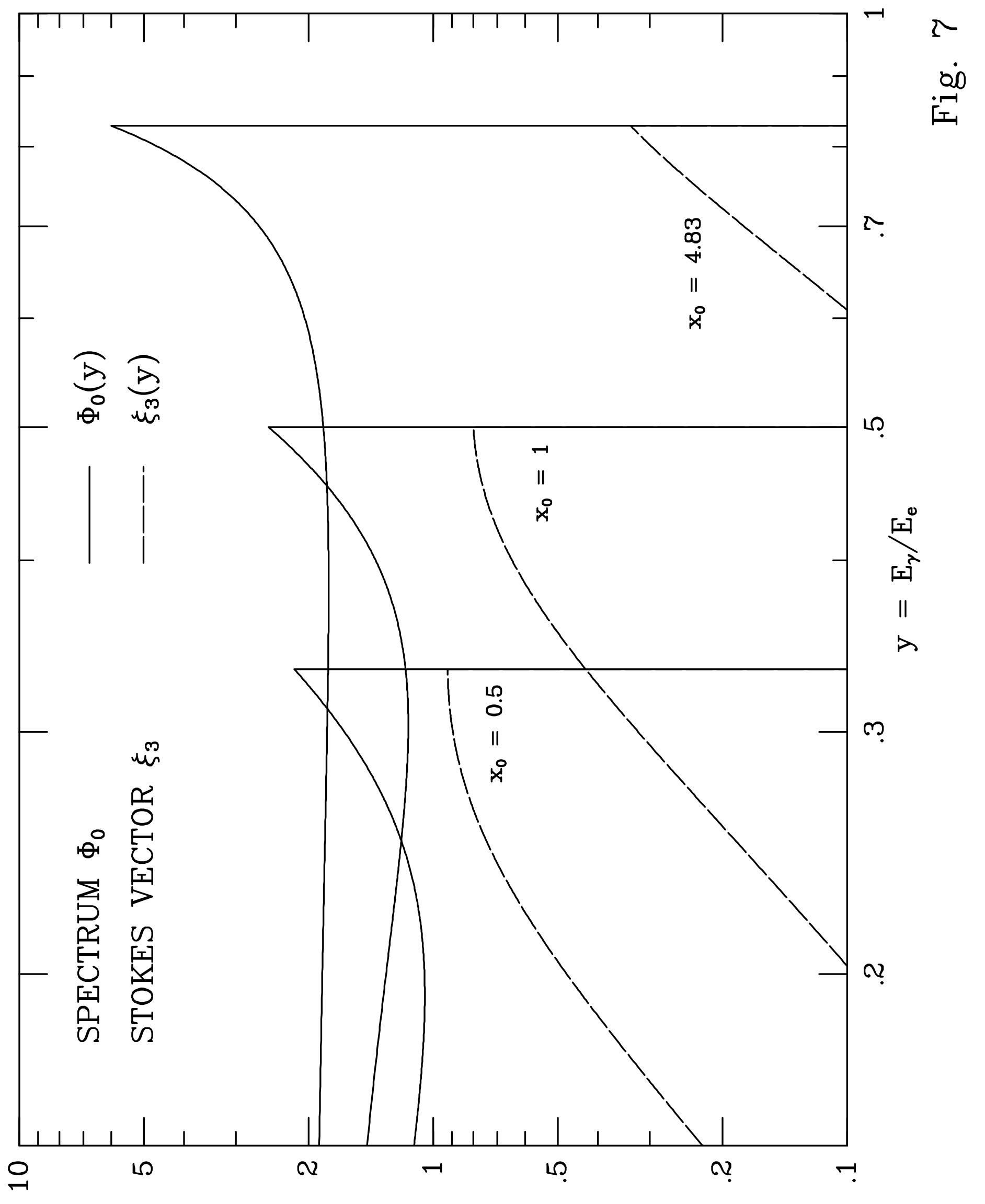




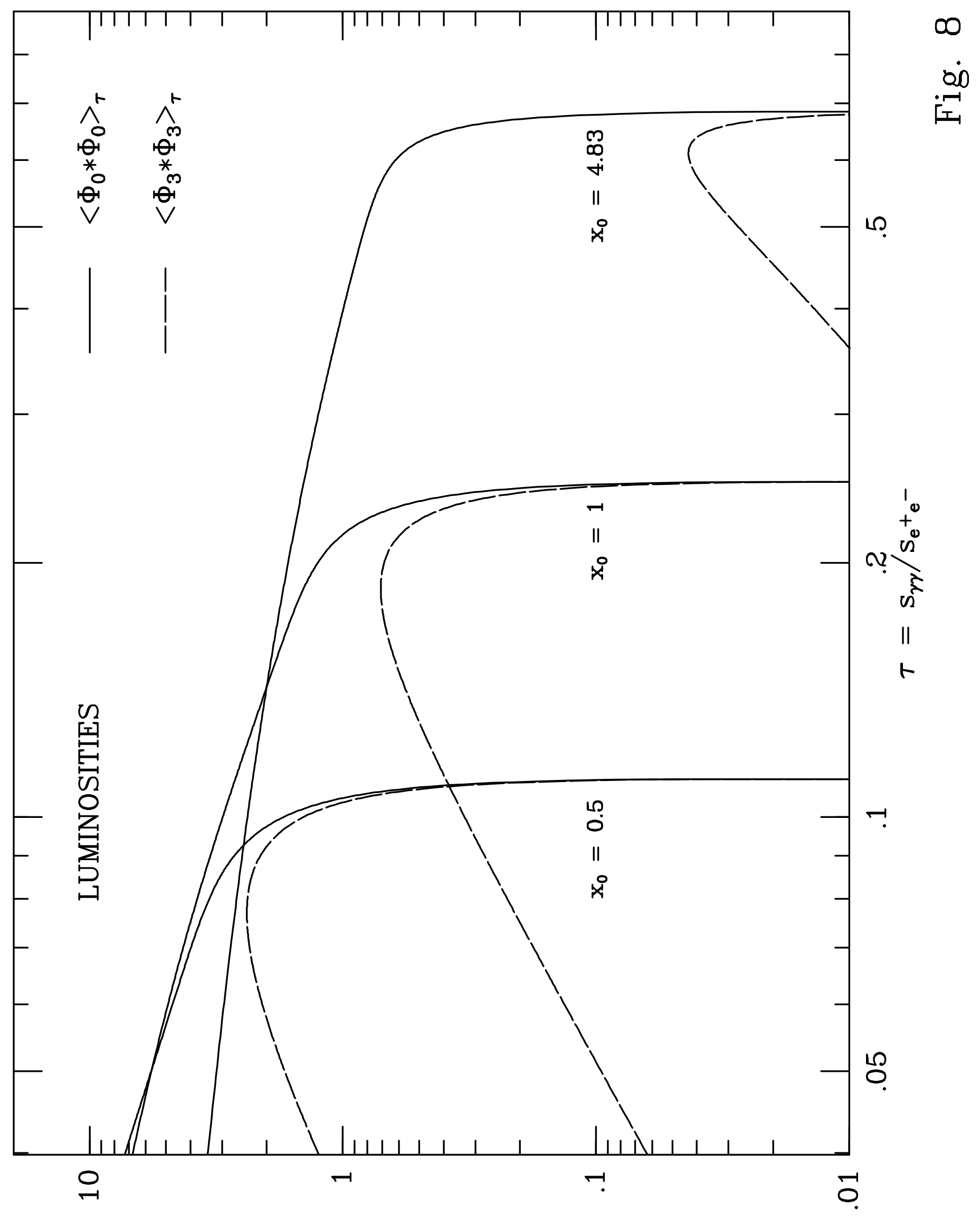




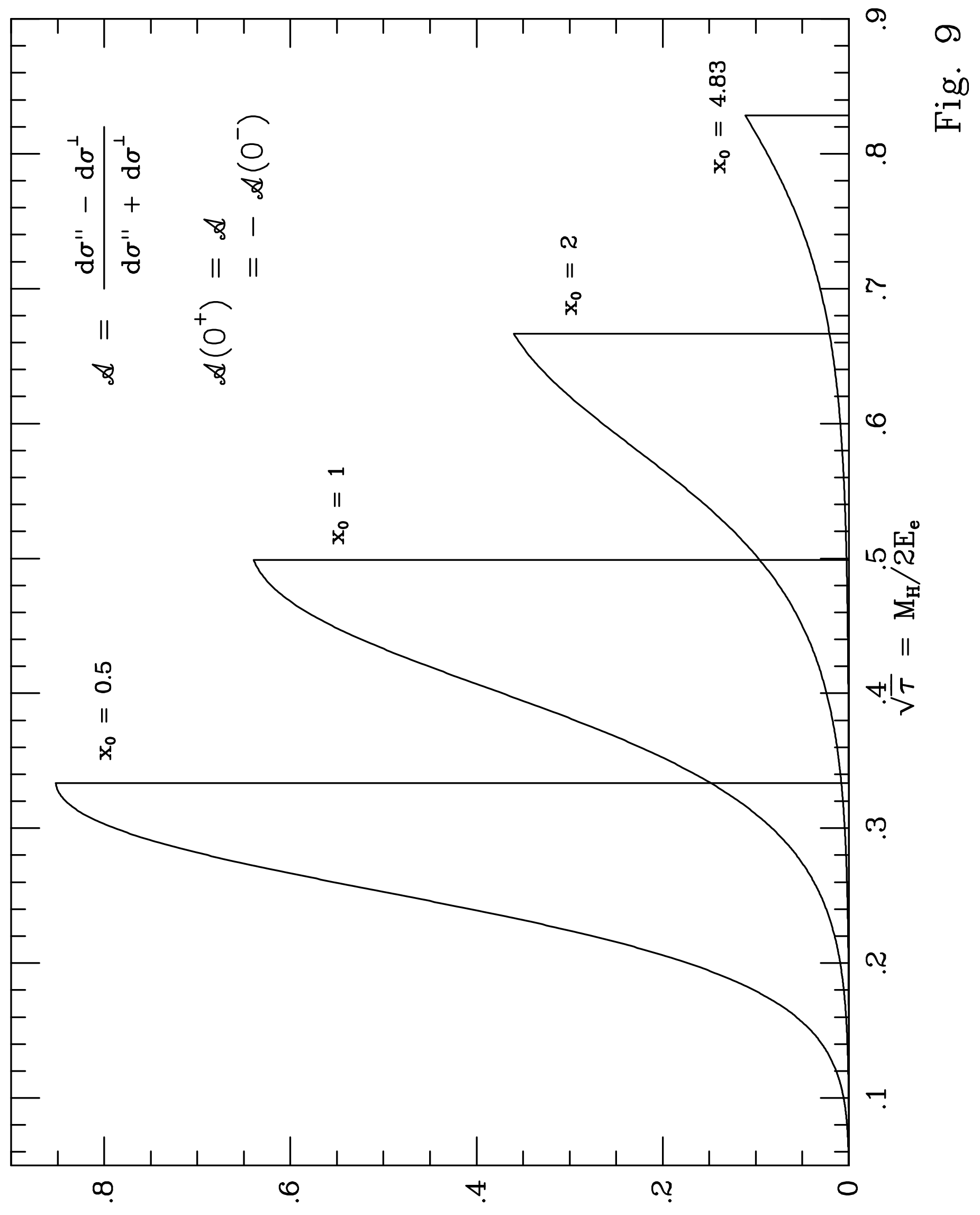




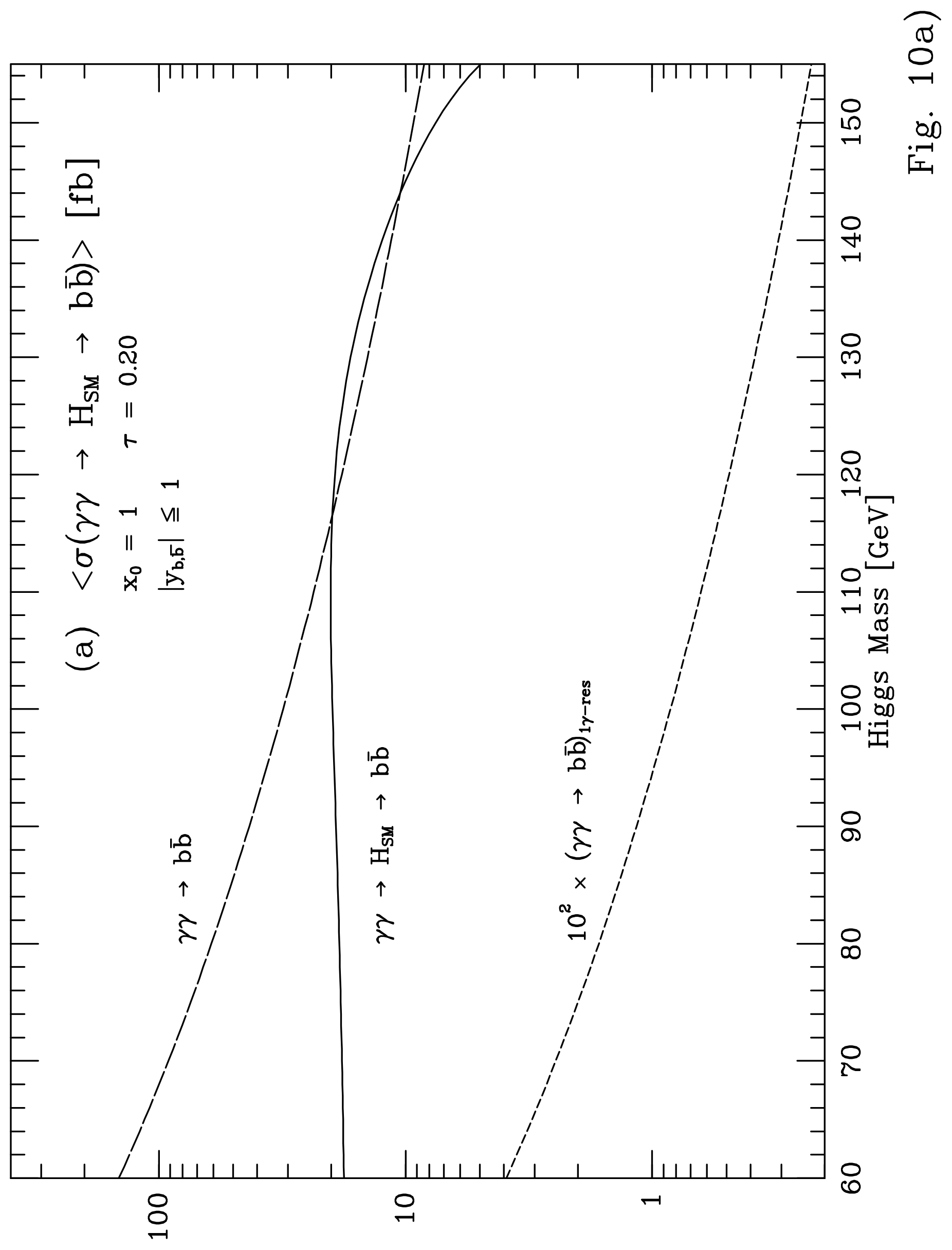




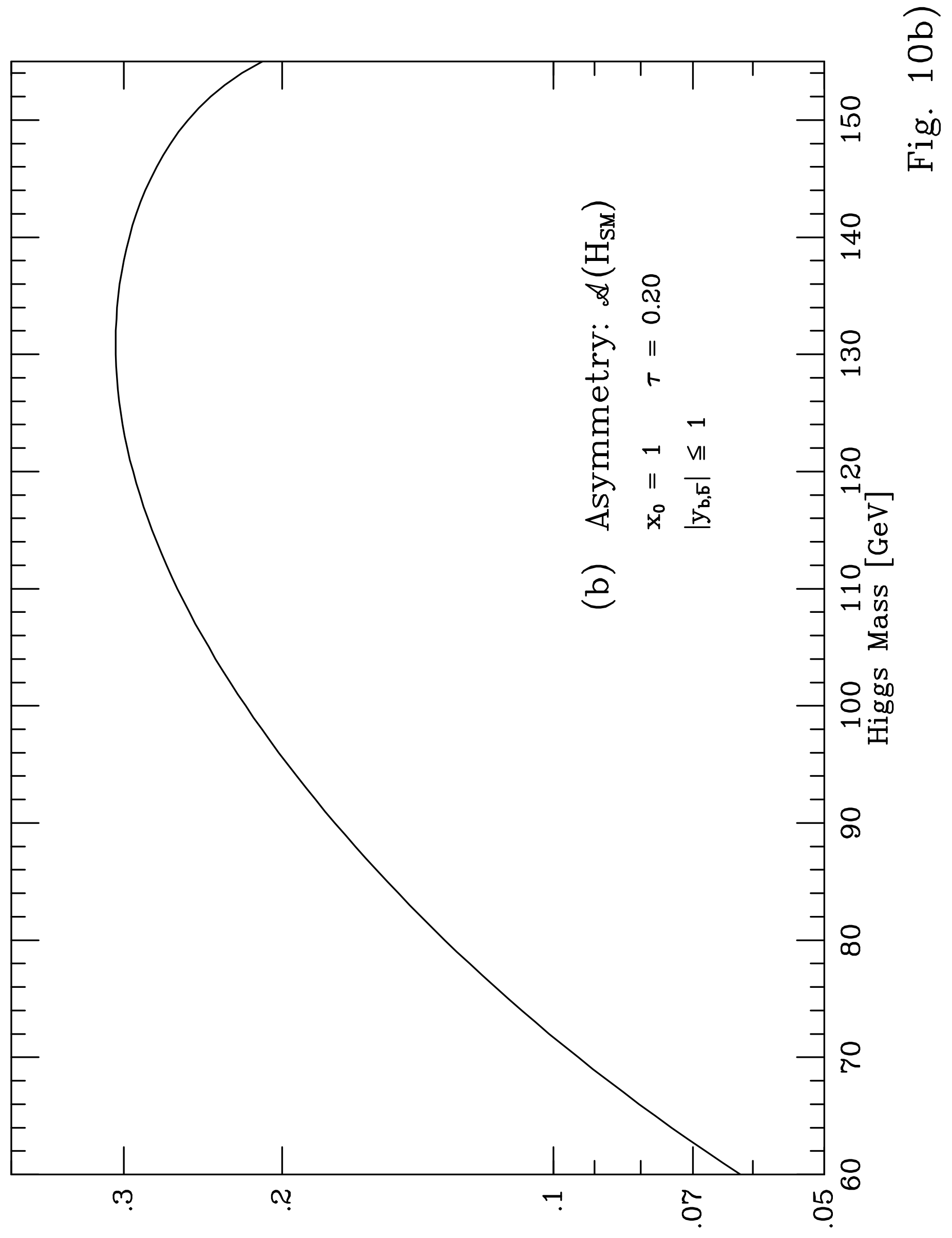




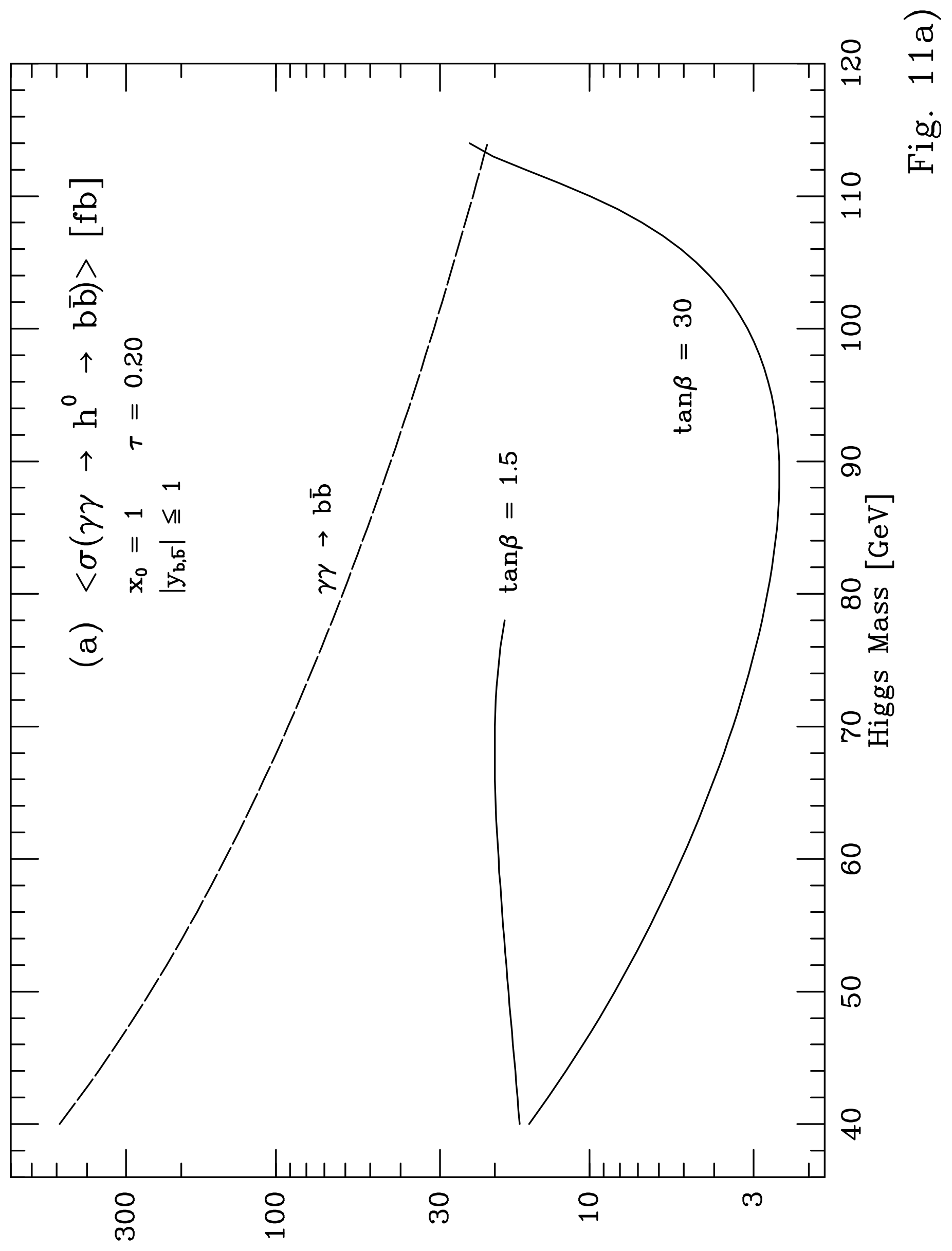




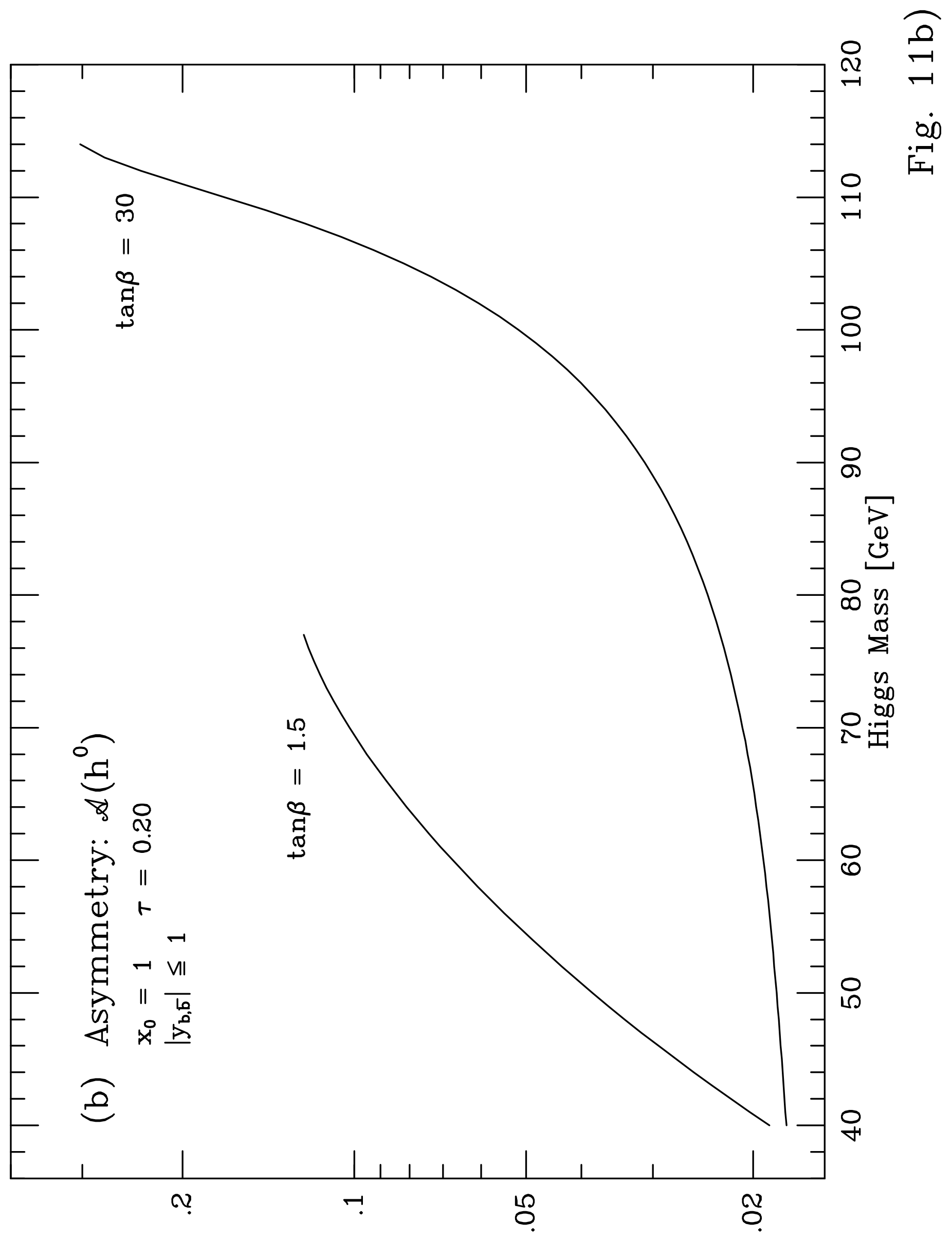




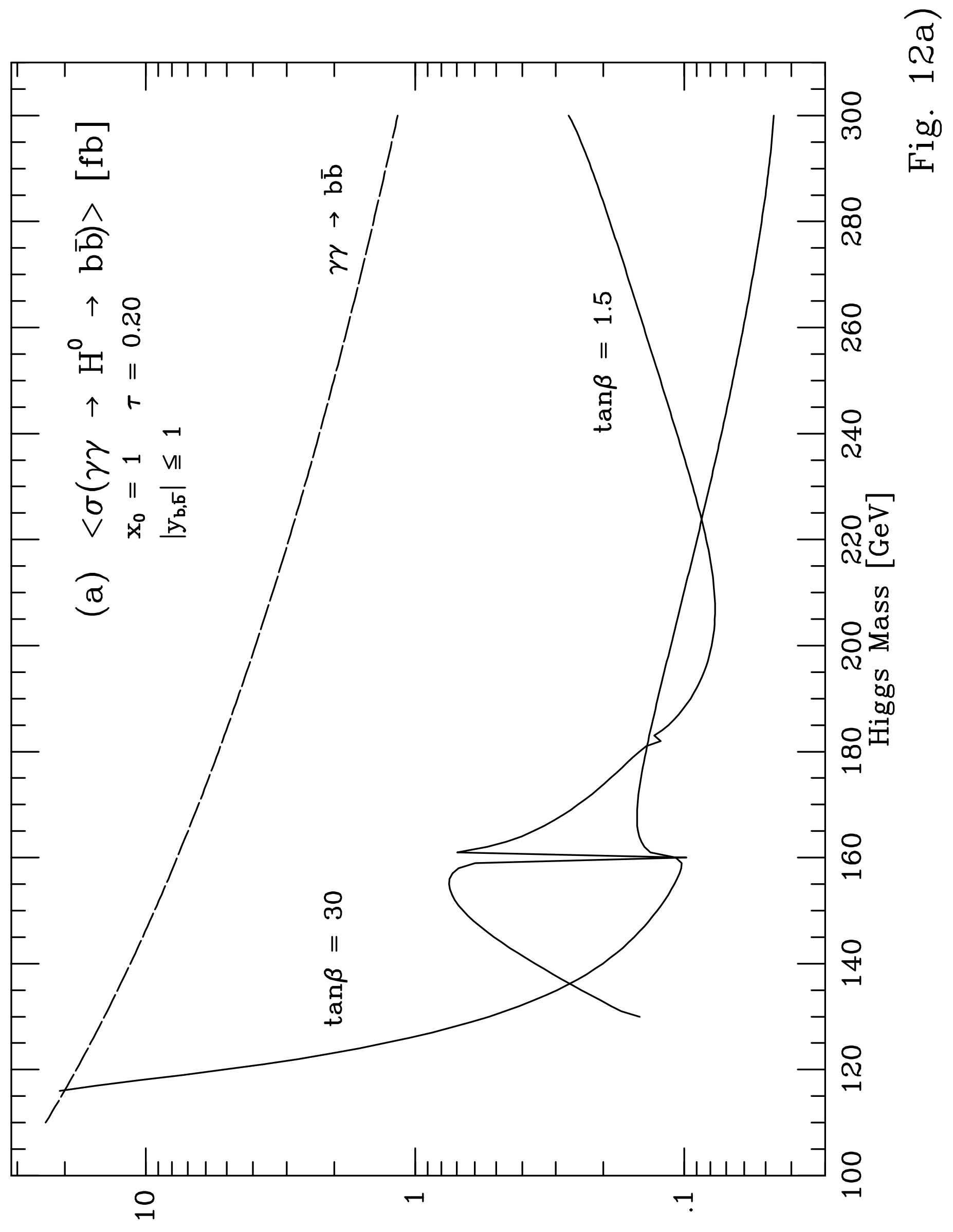




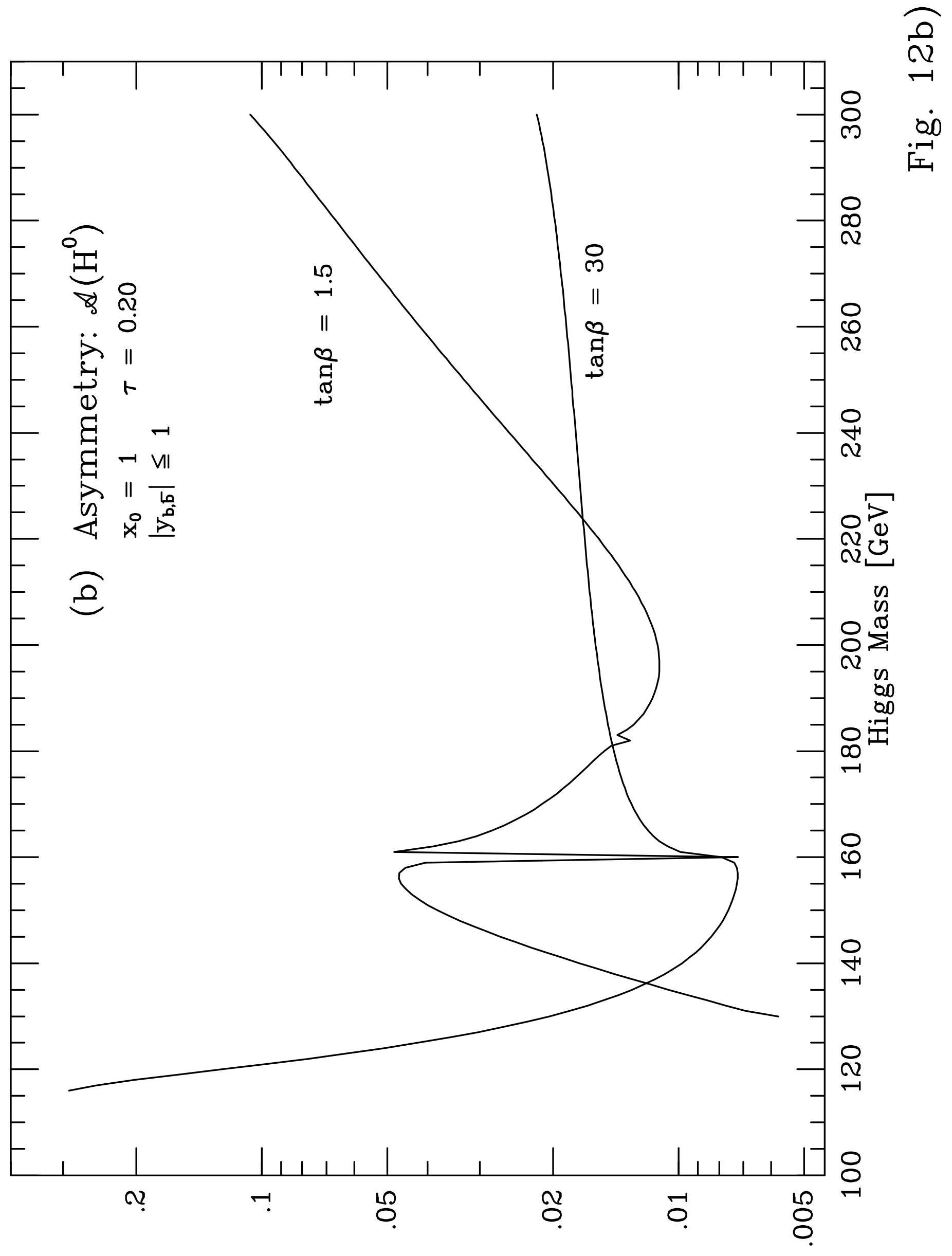




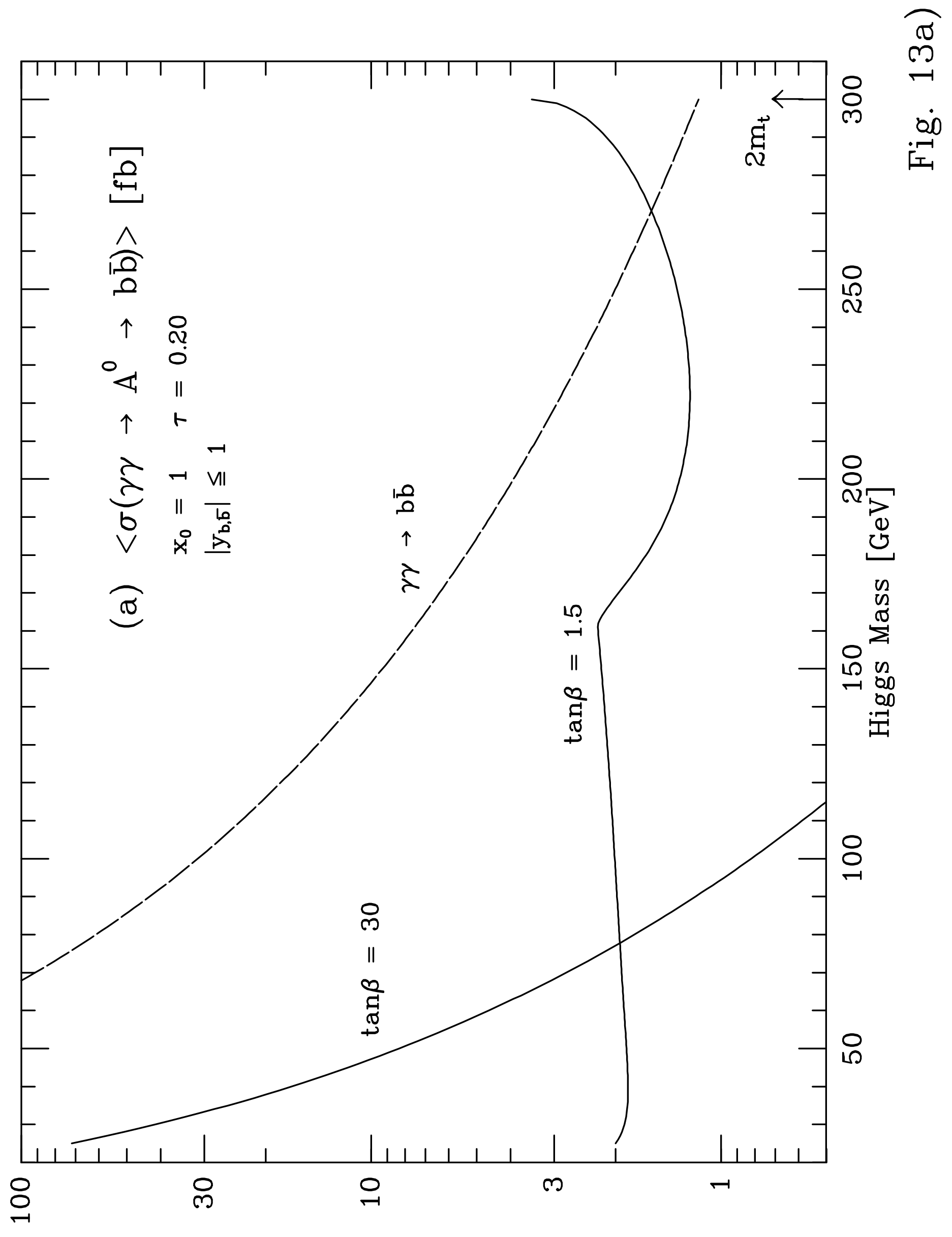




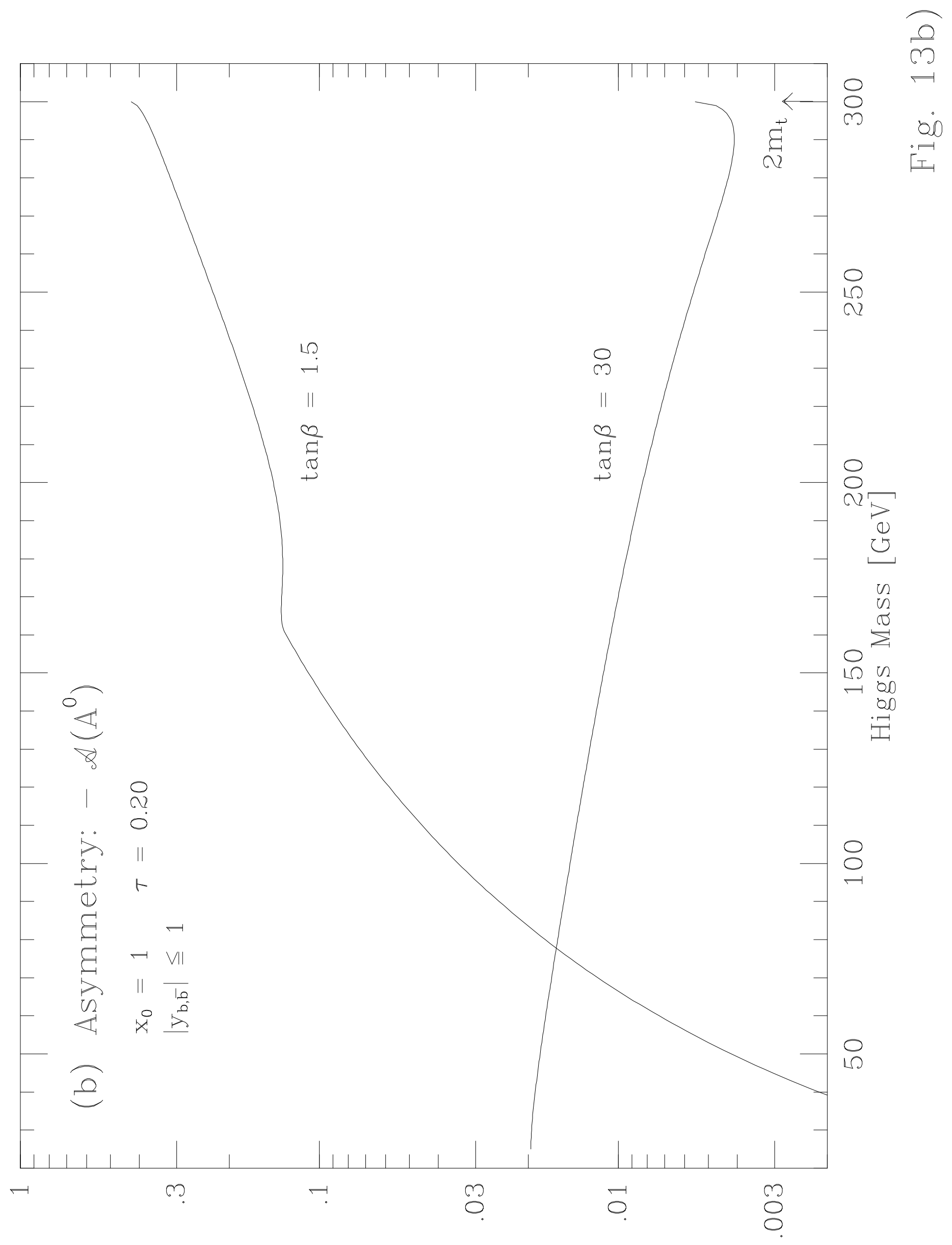

